\title{
Space-Time Joint Interference Cancellation Using Fuzzy-Inference-Based Adaptive Filtering Techniques in Frequency-Selective Multipath Channels
}

\author{
Chia-Chang Hu, ${ }^{1}$ Hsuan-Yu Lin, ${ }^{1}$ Yu-Fan Chen, ${ }^{2}$ and Jyh-Horng Wen ${ }^{1,3}$ \\ ${ }^{1}$ Department of Electrical Engineering, National Chung Cheng University, Min-Hsiung, Chia-Yi 621, Taiwan \\ ${ }^{2}$ Department of Communications Engineering, National Chung Cheng University, Min-Hsiung, Chia-Yi 621, Taiwan \\ ${ }^{3}$ Institute of Communication Engineering, National Chi Nan University, Puli, Nantou 545, Taiwan
}

Received 7 March 2005; Revised 29 May 2005; Accepted 19 July 2005

Recommended for Publication by Helmut Bolcskei

An adaptive minimum mean-square error (MMSE) array receiver based on the fuzzy-logic recursive least-squares (RLS) algorithm is developed for asynchronous DS-CDMA interference suppression in the presence of frequency-selective multipath fading. This receiver employs a fuzzy-logic control mechanism to perform the nonlinear mapping of the squared error and squared error variation, denoted by $\left(e^{2}, \Delta e^{2}\right)$, into a forgetting factor $\lambda$. For the real-time applicability, a computationally efficient version of the proposed receiver is derived based on the least-mean-square (LMS) algorithm using the fuzzy-inference-controlled step-size $\mu$. This receiver is capable of providing both fast convergence/tracking capability as well as small steady-state misadjustment as compared with conventional LMS- and RLS-based MMSE DS-CDMA receivers. Simulations show that the fuzzy-logic LMS and RLS algorithms outperform, respectively, other variable step-size LMS (VSS-LMS) and variable forgetting factor RLS (VFF-RLS) algorithms at least $3 \mathrm{~dB}$ and $1.5 \mathrm{~dB}$ in bit-error-rate (BER) for multipath fading channels.

Copyright () 2006 Hindawi Publishing Corporation. All rights reserved.

\section{INTRODUCTION}

Direct-sequence code-division multiple access (DS-CDMA), a specific form of spread-spectrum transmission, has been adopted as the multiaccess technology for nonorthogonal transmission in the third-generation $(3 \mathrm{G})$ mobile cellular systems, such as wideband CDMA (W-CDMA) or multicarrier CDMA (MC-CDMA). This sort of the code-division multiaccess techniques requires no time or frequency coordination among the mobile stations. However, the so-called near-far problem and the multipath fading are the major impediments to maintain reliable communication links in CDMA systems.

It is well known that an adaptive minimum mean-square error (MMSE) linear receiver [1] has immunity to the nearfar problem and the interference floor in performance exhibited by the conventional matched filter reception. In addition, a linear MMSE receiver can be implemented as an adaptive tapped delay line (TDL), analogous to a linear equalizer, with a relatively low complexity. However, the computation of the MMSE solution involves the calculation of the inverse of the input autocorrelation matrix, which costs a complexity of $\mathcal{O}\left((M N)^{3}\right)$. Here $M$ denotes the size of the MMSE receiving array and $N$ is the processing gain of the CDMA system so that $M N$ indicates the number of tap weights of the linear MMSE filter. This cost is even more expensive when the linear MMSE receiver operates in a nonstationary multipath environment. In practice, the filter-coefficient vector of the MMSE-type receiver can be obtained from the training sequence and the received signal by means of conventional adaptive filtering techniques, such as the least-meansquare (LMS) [2] and the recursive least-squares (RLS) [3] approaches. The LMS provides simple implementation but suffers from slow convergence, while, on the other hand, the RLS converges much faster as compared with the LMS, but it possesses more computational complexity. The drawback of slow convergence of an LMS-based algorithm, due to its dependence on the eigenvalue spread, is overcome in an RLS algorithm [3] by replacing the gradient step-size $\mu$ with a gain matrix, denoted by $\mathbf{R}_{\mathbf{x}}^{-1}[n]$, at the $n$th iteration. In [4], 
Honig et al. proposed an adaptive blind LMS implementation of the MMSE-type receiver based on the concept of the constrained minimum output energy (CMOE) for multiuser detection. An adaptive blind RLS version of the MMSE receiver was presented in [5] by Poor and Wang In [5], the proposed rotation-based QR-RLS algorithms for both the blind adaptation mode and the decision-directed adaptation mode were developed and implemented efficiently.

In recent years, the concept of fuzzy logic is used in many different senses. Fuzzy logic can be treated as a tool for embedding structured human knowledge into workable algorithms. In a wider sense, fuzzy logic is a fuzzy set theory of classes with unsharp or fuzzy boundaries. Systems designed and developed utilizing fuzzy-logic methods have been shown to be more efficient than those based on conventional approaches [6]. Notably, a fuzzy-logic controller (FLC) has been applied successfully to the fuzzy-neural scheme for on-line system identification [7] and the strength-based power control strategy in wireless multimedia cellular systems $[8,9]$. In principle, FLC provides an adaptation mechanism that converts the linguistic control strategy based on the characteristics of mobile radio channels into an adaptive parameter-control strategy. By using the defuzzification, the fuzzy control decisions are converted to a crisp control command which is used to adjust properly the level of the parameter of interest. To improve the FLC performance, the use of a fuzzy proportional-plus-integral (PI) control is addressed in [10].

In the present paper, an adaptive robust MMSE arrayreceiver is proposed based on a fuzzy-logic controlled LMS or RLS algorithm for space-time joint asynchronous DS-CDMA signals. The FLC system is employed to perform the nonlinear mapping of the input variables into a scalar adaptation step-size $\mu$ of the LMS algorithm or a forgetting factor $\lambda$ of the RLS algorithm in response to the channel variation. Note that the input variables of the FLC system may include the error signal, duration of training, squared error, input power, and any other useful variables. Owing to the flexibility and richness of the fuzzy-inference control system, it may produce many different mappings that are especially suitable for applications in nonlinear and time-varying cellular systems. In [11], the behavior of different adaptive LMS algorithms with the fuzzy step size is analyzed. Experimental results show that the fuzzy step-size LMS (FSS-LMS) algorithms proposed by the author in [11] possess superior convergence characteristics than other existing variable step-size LMS (VSS-LMS) approaches $[12,13]$. In particular, the performance of the FSS-LMS system with two inputs of $e^{2}$ and $N_{T}$ is noticeable, where $e^{2}$ is the squared error and $N_{T}$ denotes the duration of training. Unfortunately, the quantity of $N_{T}$ may not be attainable to the category of adaptive blindbased receivers. In [14], the authors proposed the variable forgetting factor linear least-squares (VFF-LLS) algorithm to improve the tracking capability of channel estimation. These works motivate the development of the linear MMSE CDMA receiver with a fuzzy-logic controlled two-parameter system of $\left(e^{2}, \Delta e^{2}\right)$ instead of $\left(e^{2}, N_{T}\right)$, where $\Delta e^{2}[n] \triangleq \mid e^{2}[n]-e^{2}[n-$ $1] \mid$ indicates the squared error variation at time $n$. In other words, the pair values of $\left(e^{2}, \Delta e^{2}\right)$ are calculated and fed to the FLC system to assign an exact value of $\mu$ or $\lambda$ for the corresponding adaptive receiver on an iterative basis in order to improve the convergence characteristic and steady-state MSE simultaneously. Most of the fuzzy inference rules are derived by a human expert or extracted from numerical data. In this paper, we focus attention on the fuzzy rules which accumulate past experience operating in the practical applications. Therefore, it seems natural and reasonable to expect that wireless communication systems with the use of a twoparameter $\left(e^{2}, \Delta e^{2}\right)$-FLC produce better convergence characteristics than those with only single-parameter $\left(e^{2}\right)$-FLC. Furthermore, the pair of $\left(e^{2}, \Delta e^{2}\right)$ provides the FLC system with more precise channel dynamic-tracking and adaptation capability than the pair of $\left(e^{2}, N_{T}\right)$. This is because the "auxiliary" parameter $\Delta e^{2}$ offers an effective and robust means to monitor instantaneous fluctuations of a fast-fading multipath channel and assists the FLC system in selecting an appropriate value for $\mu$ or $\lambda$. It is remarkable that the proposed FLC-based approaches produce a faster speed of convergence without trading off the steady-state performance.

Computational requirements of the proposed fuzzylogic-controlled LMS and RLS algorithms of the MMSE receiver, abbreviated as FLC-LMS and FLC-RLS hereafter, are evaluated in this paper. Slightly additional computational load is incurred in the fuzzification (table lookup), inference (MIN, MAX, and PROD operators), and defuzzification processes. There is also additional cost which comes from the preparation of the two input variables, $\left(e^{2}, \Delta e^{2}\right)$, prior to the fuzzification process. Fortunately, these operations can be done very efficiently in the latest range of DSPs which provide single-cycle multiply and add, table lookup, and comparison instructions. The computational load is compared to other known MMSE CDMA algorithms as well.

The material included in this paper is organized as follows. In Section 2, an asynchronous DS-CDMA signal model is outlined. For demodulation, an adaptive linear MMSE array receiver is employed and implemented by the fuzzy-logic controlled LMS and RLS algorithms. The proposed MMSE CDMA receiver is developed in Section 3. Section 3.1 describes briefly the ideal MMSE solution for DS-CDMA interference suppression. In Section 3.2, the FLC-LMS and FLCRLS algorithms of the MMSE CDMA receiver are derived in detail. Section 3.3 presents the analysis to compare the computational load of the proposed algorithms with other equivalent DS-CDMA schemes. In Section 4, a brief review of existing VSS-LMS and VFF-RLS algorithms is provided. The convergence/tracking capability and the steady-state performance of the proposed MMSE receiver under the frequencyselective multipath fading channel is analyzed in Section 5. Finally, concluding remarks are given in Section 6.

\section{SIGNAL MODEL}

An asynchronous DS-CDMA system operating over a dynamic fading multipath channel is considered. The transmitted baseband signal $r_{l}(t)$ for user $l$ is obtained by spreading a set of BPSK data symbols $\left\{d_{l}[i]\right\}$, that is, a set of independent 
equiprobable \pm 1 random variables, onto a spreading waveform $s_{l}(t)$. That is,

$$
r_{l}(t)=\sum_{i=-\infty}^{\infty} \sqrt{E_{l}} d_{l}[i] s_{l}\left(t-i T_{b}\right),
$$

where $E_{l}$ denotes bit energy of user $l$. The spreading waveform $s_{l}(t)$ is generated by modulating a spreading sequence $c_{l, k} \in\{-1,1\}, k=0,1, \ldots, N-1$, of length $N$ with a train of rectangular chip waveforms, $\psi(t)$, of duration $T_{c}=T_{b} / N$, that is,

$$
s_{l}(t)=\sum_{k=0}^{N-1} c_{l, k} \psi\left(t-k T_{c}\right), \quad t \in\left[0, T_{b}\right),
$$

where $T_{b}$ is the bit interval. In [15], a multipath fading channel of user $l$ can be described by its baseband complex impulse response

$$
h_{l}(t)=\sum_{j=1}^{K_{l}} a_{l j} \delta\left(t-\tau_{l j}\right)
$$

where $K_{l}$ denotes the total number of distinct, resolvable, propagation paths of user $l$. In this paper, $K_{l}$ is set equally for all users. Here $\delta(\cdot)$ denotes the Dirac delta function, $a_{l j}$ is the complex channel fading coefficient, and $\tau_{l j}$ is the propagation delay, which are associated with the $j$ th propagation path of user $l$. To model frequency-selective fading, multipath components are assumed to fade independently [16]. The discretized sequence of channel coefficients for the lth user is a complex Gaussian random process obtained by passing complex white Gaussian noise through a filter with transfer function $\kappa / \sqrt{1-\left(f / f_{D}\right)^{2}}$, where $\kappa=1 / \pi f_{D}$ is a normalization constant, $f_{D}=v / \lambda$ is the maximum Doppler frequency, and $v$ is the user's vehicle speed. In general, the value of the normalized fading rate, $f_{D} T_{b}$, that is, the product of the Doppler frequency and the symbol period, determines the degree of signal fading. For slowly fading channels, $f_{D} T_{b} \ll 1$. In other words, the transmitted signal of the $l$ th user is distorted by a frequency-selective multipath channel, modeled in discrete time by an $K_{l}$-tap tapped delay line (TDL) whose coefficients are represented by $\mathbf{h}_{l}[n]=\left[h_{l 1}[n]\right.$, $\left.h_{l 2}[n], \ldots, h_{l K_{l}}[n]\right]$, where $K_{l}$ is known as the delay spread of the channel. The multipath spread of the channel is assumed to be less than one symbol period in this paper. The channel coefficients of user $l, h_{l j}[n], j=1,2, \ldots, K_{l}$, are independent random variables with Rayleigh distribution. Independent fading on each path implies that $h_{l j}[n]$ and $h_{l k}[n]$, $j \neq k$, are independent for all $n$.

After multipath fading channel "processing," the total received signal at the receiver is a superposition of propagated signals from all $K$ users and the background channel noise. The received signal $\mathbf{r}(t)$ can be written as

$$
\begin{aligned}
\mathbf{r}(t) & =\sum_{l=1}^{K} \mathbf{r}_{l}(t)+\mathbf{n}(t) \\
& =\sum_{l=1}^{K} \sqrt{E_{l}} \sum_{j=1}^{K_{l}} a_{l j} \mathbf{b}_{l j} \sum_{i=-\infty}^{\infty} d_{l}[i] s_{l}\left(t-i T_{b}-\tau_{l j}\right)+\mathbf{n}(t),
\end{aligned}
$$

where $\mathbf{n}(t)$ is an additive white Gaussian noise (AWGN) vector. The $M \times 1$ linear array response vector $\mathbf{b}_{l j}$ for the $j$ th path of the $l$ th user's signal is defined by $b_{l j}^{m}=e^{j 2 \pi(m-1)(d / \lambda) \sin \theta_{l j}}$, $m=1,2, \ldots, M$, where $\lambda$ is the carrier wavelength, $d$ denotes the element spacing of the antenna, and $\theta_{l j}$ identifies the angle of arrival (AOA). In addition, it is assumed that all channels are constant during each symbol period and the receiver's clock is synchronized with the reception of the first path of the desired user, say of user 1 , that is, $\tau_{11}=0$ [17]. Note that the term asynchronous means that the timings of signals and multipaths from different users received by the base station in either intracell or intercell are not the same. In other words, the propagation delays associated with the propagation paths of different users are considered in this paper.

\section{RECEIVER ARCHITECTURE}

For convenience, the proposed receiver is described by means of a baseband-equivalent structure. The received signal of each individual antenna element is passed through a filter that is matched to the square-wave chip waveform. If $r_{m}(t)$ is the $m$ th component of $\mathbf{r}(t)$ in (4), the output of the $m$ th antenna element is

$$
z_{m}(t)=\int_{-\infty}^{t} \psi\left(t-t^{\prime}\right) r_{m}\left(t^{\prime}\right) d t^{\prime}=\int_{0}^{T_{c}} r_{m}(t-u) d u,
$$

for $m=1,2, \ldots, M$. Subsequently, the output of this chip matched filter $(\mathrm{MF})$ is sampled at the chip rate $1 / T_{c}$ over the multipath extended $\left(N+K_{l}-1\right)$-chip period for one-shot data detection. These discrete-time outputs are used as the inputs of $M$ adaptive, $\left(N+K_{l}-1\right)$-element TDLs with a tap spacing of $T_{c}$ to form $M$ such $\left(N+K_{l}-1\right)$-element data vectors. Assume that the output signals of the chip MFs are sampled at the times $n T_{c}$. The TDLs for the $M$-element antenna array are expressed as an $M \times\left(N+K_{l}-1\right)$ data array, given by

$$
\begin{aligned}
& \mathbf{Z}[n] \\
& =\left[\begin{array}{cccc}
z_{1}\left(n T_{c}\right) & z_{1}\left((n-1) T_{c}\right) & \cdots & z_{1}\left(\left(n-N-K_{l}+2\right) T_{c}\right) \\
z_{2}\left(n T_{c}\right) & z_{2}\left((n-1) T_{c}\right) & \cdots & z_{2}\left(\left(n-N-K_{l}+2\right) T_{c}\right) \\
\vdots & \vdots & \ddots & \vdots \\
z_{M}\left(n T_{c}\right) & z_{M}\left((n-1) T_{c}\right) & \cdots & z_{M}\left(\left(n-N-K_{l}+2\right) T_{c}\right)
\end{array}\right] .
\end{aligned}
$$

The data matrix $\mathbf{Z}[n]$ is then "vectorized" by sequencing all matrix rows to form the $M\left(N+K_{l}-1\right)$ vector as follows:

$$
\mathbf{x}[n]=\operatorname{Vec}\{\mathbf{Z}[n]\}=\left[x_{1}[n], x_{2}[n], \ldots, x_{M\left(N+K_{l}-1\right)}[n]\right]^{\mathrm{T}} \text {. }
$$

The symbol $(\cdot)^{\mathrm{T}}$ denotes matrix transpose. The vector $\mathbf{x}[n]$ in (7) denotes the joint space-time data of the $\mathrm{C}^{M \times\left(N+K_{l}-1\right)}$ complex vector domain, and the $x_{i}[n]$ for $i=$ $1,2, \ldots, M\left(N+K_{l}-1\right)$ are the data components of the vector $\mathbf{x}[n]$, lexigraphically ordered. Similarly, the adaptive 
weight vector of a filter for vector $\mathbf{x}[n]$ is expressed as the column vector,

$$
\mathbf{w}[n]=\left[w_{1}[n], w_{2}[n], \ldots, w_{M\left(N+K_{l}-1\right)}[n]\right]^{\mathrm{T}} .
$$

The components of the weight vector $\mathbf{w}[n] \in \mathcal{C}^{M\left(N+K_{l}-1\right) \times 1}$ are adapted to minimize the MSE at the output of the TDLs (i.e., an MMSE-type filter coefficients) and determined explicitly later in (16) and (21). The output of the tapped delayline adaptive filter for $\mathbf{x}[n]$ is the inner product of the vectors in (7) and (8) as follows:

$$
y[n]=\mathbf{w}^{\dagger}[n] \mathbf{x}[n]=\sum_{i=1}^{M\left(N+K_{l}-1\right)} w_{i}^{*}[n] x_{i}[n]
$$

where the superscripts $(\cdot)^{\dagger}$ and $(\cdot)^{*}$ denote the conjugate transpose (Hermitian) of a matrix and the conjugate of a complex number, respectively.

\subsection{MMSE demodulator}

In [1], the minimum mean-square error (MMSE) linear equalizer for asynchronous CDMA systems was first proposed by Xie et al. as a nonadaptive receiver. This was followed by various adaptive recursive implementations which operated in a decision-directed (DD) mode [18-20]. Later it was shown in [4] that the linear MMSE receiver can be operated in a blind adaptation manner which obviates the necessity of training. The MMSE receiver often is employed to detect the desired information symbol, owing to its simple implementation and excellent performance. Let $\mathbf{x}[n]$ denote the observation vector obtained at time clock $n$. The linear MMSE receiver has the form

$$
\hat{d}_{1}[n]=\operatorname{sgn}\left(\operatorname{Re}\left\{\mathbf{w}^{\dagger}[n] \mathbf{x}[n]\right\}\right),
$$

where sgn denotes the sign operator, $\operatorname{Re}\{\cdot\}$ takes the real part, and $\mathbf{x}[n]$ is given by (7). The weight vector $\mathbf{w}[n] \in$ $\mathrm{C}^{M\left(N+K_{l}-1\right) \times 1}$ is chosen to minimize the MSE,

$$
\operatorname{MSE}[n] \triangleq E\left\{|e[n]|^{2}\right\}
$$

where the output error $e[n]$ between the decision statistic and the transmitted symbol is expressed by

$$
e[n]=d_{1}[n]-y[n]=d_{1}[n]-\mathbf{w}^{\dagger}[n] \mathbf{x}[n] .
$$

It is easy to show that the ideal MMSE solution of the weight vector $\mathbf{w}[n]$ is given by the vector

$$
\mathbf{w}_{\operatorname{MMSE}[n]}=E\left\{\mathbf{x}[n] \mathbf{x}^{\dagger}[n]\right\}^{-1} E\left\{d_{1}^{*}[n] \mathbf{x}[n]\right\}=\mathbf{R}_{\mathbf{x}}^{-1}[n] \mathbf{p}_{1}[n],
$$

where $\mathbf{R}_{\mathbf{x}}[n]=E\left\{\mathbf{x}[n] \mathbf{x}^{\dagger}[n]\right\}$ and $\mathbf{p}_{1}[n]=E\left\{d_{1}^{*}[n] \mathbf{x}[n]\right\}$ are the input autocorrelation matrix and the steering vector, that is, the result of correlating the desired bit with the observation vectors, respectively. The notation $E\{\cdot\}$ denotes the expected-value operator. The MSE achieved by the MMSE solution in (13) is given by

$$
\begin{aligned}
\operatorname{MMSE}[n] & =\min _{\mathbf{w}[n]} \operatorname{MSE}[n]=1-\mathbf{p}_{1}^{\dagger}[n] \mathbf{R}_{\mathbf{x}}^{-1}[n] \mathbf{p}_{1}[n] \\
& =1-\mathbf{w}_{\operatorname{MMSE}}^{\dagger}[n] \mathbf{p}_{1}[n]
\end{aligned}
$$

Then the estimate of the information symbol $d_{1}[n]$ is obtained from the expression

$$
\hat{d}_{1}[n]=\operatorname{sgn}\left(\operatorname{Re}\left\{\mathbf{p}_{1}^{\dagger}[n] \mathbf{R}_{\mathbf{x}}^{-1}[n] \mathbf{x}[n]\right\}\right) .
$$

In practice, an adaptive linear MMSE demodulator is usually achieved by means of training with respect to a known training or pilot sequence $\left\{d_{1}[k]\right\}_{k=1}^{N_{T}}$ of length $N_{T}$, followed by a DD adaptation utilizing the estimate symbol $\hat{d}_{1}$ as the feedback information for better adaptation. The adaptive implementation can be realized using a variety of well-known algorithms, for example, stochastic gradient (SG), least squares (LS), and recursive least-squares (RLS). In this paper, the adaptive implementations of the LMS and the RLS for the proposed MMSE demodulator are described in detail as follows:

\section{LMS adaptation}

An adaptive LMS-type filter calculates the estimates of the receiver tap-weight vector by minimizing the MSE in (11), that is, $E\left\{\left|d_{1}[n]-\mathbf{w}^{\dagger}[n] \mathbf{x}[n]\right|^{2}\right\}$. The tap-weight estimate at the $(n+1)$ th iteration using information available up to the iteration $n$ is

$$
\mathbf{w}[n+1]=\mathbf{w}[n]+\mu\left(d_{1}^{*}[n]-\mathbf{x}^{\dagger}[n] \mathbf{w}[n]\right) \mathbf{x}[n],
$$

where the positive scalar $\mu$ denotes the step size of the LMS algorithm, which depends on the statistics of the observation vector $\mathbf{x}[n]$. For stability, $\mu$ needs to be implicitly bounded in magnitude by the values of its minimum ( $\mu_{\min }=0$ or the smallest possible value) and maximum $\left(\mu_{\max } \triangleq \min _{k}\left(2 /\left|\alpha_{k}\right|\right)\right.$, where $\alpha_{k}$ stands for the $k$ th eigenvalue of $\left.\mathbf{R}_{\mathbf{x}}[n]\right)$ values.

\section{RLS adaptation}

The convergence rate of the LMS algorithm depends principally upon the eigenvalue spread of $\mathbf{R}_{\mathbf{X}}[n]$. In an environment yielding $\mathbf{R}_{\mathbf{X}}[n]$ with a large eigenvalue spread, the LMS algorithm converges with a slow speed. This problem is solved in an RLS algorithm by replacing the gradient step-size $\mu$ with a gain matrix $\mathbf{R}_{\mathbf{x}}^{-1}[n]$ at iteration $n$, producing the weight update equation

$$
\mathbf{w}[n]=\mathbf{w}[n-1]+\mathbf{R}_{\mathbf{x}}^{-1}[n]\left(d_{1}^{*}[n]-\mathbf{x}^{\dagger}[n] \mathbf{w}[n-1]\right) \mathbf{x}[n],
$$

with $\mathbf{R}_{\mathbf{x}}[n]$ given by

$$
\begin{aligned}
\mathbf{R}_{\mathbf{x}}[n] & =\lambda \mathbf{R}_{\mathbf{x}}[n-1]+\mathbf{x}[n] \mathbf{x}^{\dagger}[n] \\
& =\sum_{k=0}^{n} \lambda^{n-k} \mathbf{x}[k] \mathbf{x}^{\dagger}[k],
\end{aligned}
$$


where the quantity of $\lambda \in(0,1]$ is normally referred to as the exponential weighting factor, or forgetting factor, of the RLS algorithm. The reciprocal of $1-\lambda$ is a measure of the memory of the algorithm. The special case, as $\lambda$ approaches one, corresponds to infinite memory. The inverse of $\mathbf{R}_{\mathbf{x}}[n]$ required in (17) is computed by the Woodbury's identity ${ }^{1}$ [21]

$$
\mathbf{R}_{\mathbf{x}}^{-1}[n]=\lambda^{-1} \mathbf{R}_{\mathbf{x}}^{-1}[n-1]-\frac{\lambda^{-2} \mathbf{R}_{\mathbf{x}}^{-1}[n-1] \mathbf{x}[n] \mathbf{x}^{\dagger}[n] \mathbf{R}_{\mathbf{x}}^{-1}[n-1]}{1+\lambda^{-1} \mathbf{X}^{\dagger}[n] \mathbf{R}_{\mathbf{x}}^{-1}[n-1] \mathbf{x}[n]} .
$$

The matrix is usually initialized as $\mathbf{R}_{\mathbf{x}}^{-1}[0]=\delta^{-1} \mathbf{I}$ with $\delta>0$, where I is the $M\left(N+K_{l}-1\right) \times M\left(N+K_{l}-1\right)$ identity matrix.

An adaptive RLS-type filter calculates the estimates of the tap-weight vector by minimizing the cumulative exponentially-weighted squared error, that is,

$$
\sum_{k=0}^{n} \lambda^{n-k}|e[k]|^{2}=\sum_{k=0}^{n} \lambda^{n-k}\left|d_{1}[k]-\mathbf{w}^{\dagger}[k] \mathbf{x}[k]\right|^{2}
$$

By using (19), the recursive equation of the RLS algorithm in (17) for updating the tap-weight vector at iteration $n$ is reexpressed as

$$
\begin{aligned}
\mathbf{w}[n] & =\mathbf{w}[n-1]+\xi^{*}[n] \mathbf{k}[n] \\
& =\mathbf{w}[n-1]+\left(d_{1}^{*}[n]-\mathbf{x}^{\dagger}[n] \mathbf{w}[n-1]\right) \mathbf{k}[n],
\end{aligned}
$$

where the scalar $\xi[n]=d_{1}[n]-\mathbf{w}^{\dagger}[n-1] \mathbf{x}[n]$ defines a priori estimation error, which is generally different from the a posteriori estimation error $e[n]$ defined in (20), and the $M\left(N+K_{l}-1\right)$-vector $\mathbf{k}[n]$ denotes the time-varying gain vector given by

$$
\mathbf{k}[n]=\frac{\mathbf{P}[n-1] \mathbf{x}[n]}{\lambda+\mathbf{x}^{\dagger}[n] \mathbf{P}[n-1] \mathbf{x}[n]}
$$

with the $M\left(N+K_{l}-1\right) \times M\left(N+K_{l}-1\right)$ matrix $\mathbf{P}[n]$, which is defined by the inverse autocorrelation matrix $\mathbf{R}_{\mathbf{x}}^{-1}[n]$, computed by the Riccati equation as follows:

$$
\mathbf{P}[n]=\lambda^{-1} \mathbf{P}[n-1]-\lambda^{-1} \mathbf{k}[n] \mathbf{x}^{\dagger}[n] \mathbf{P}[n-1] .
$$

By rearranging (22), the fact that $\mathbf{P}[n] \mathbf{x}[n]$ equals the gain vector $\mathbf{k}[n]$ is easily verified.

\subsection{Fuzzy-inference-based LMS and RLS adaptation}

The conventional LMS-based adaptive filter uses a constant step size to update its weight coefficients in response to the changing environment. A large step size usually leads to a faster initial convergence, but results in larger fluctuation in the steady-state MSE. The opposite phenomena occur when a small step size is utilized. To overcome this problem, the decision of the step size is generally made by a tradeoff between convergence time and steady-state error.

\footnotetext{
${ }^{1}$ Woodbury's identity (or the matrix inversion lemma) $\mathbf{A}^{-1}=\left(\mathbf{B}^{-1}+\right.$ $\left.\mathbf{C D}^{-1} \mathbf{C}^{\dagger}\right)^{-1}=\mathbf{B}-\mathbf{B C}\left(\mathbf{D}+\mathbf{C}^{\dagger} \mathbf{B C}\right)^{-1} \mathbf{C}^{\dagger} \mathbf{B}$ is applied to (19) with $\mathbf{A}=$ $\mathbf{R}_{\mathbf{X}}[n], \mathbf{B}^{-1}=\lambda \mathbf{R}_{\mathbf{x}}[n-1], \mathbf{C}=\mathbf{x}[n]$, and $\mathbf{D}^{-1}=1$.
}

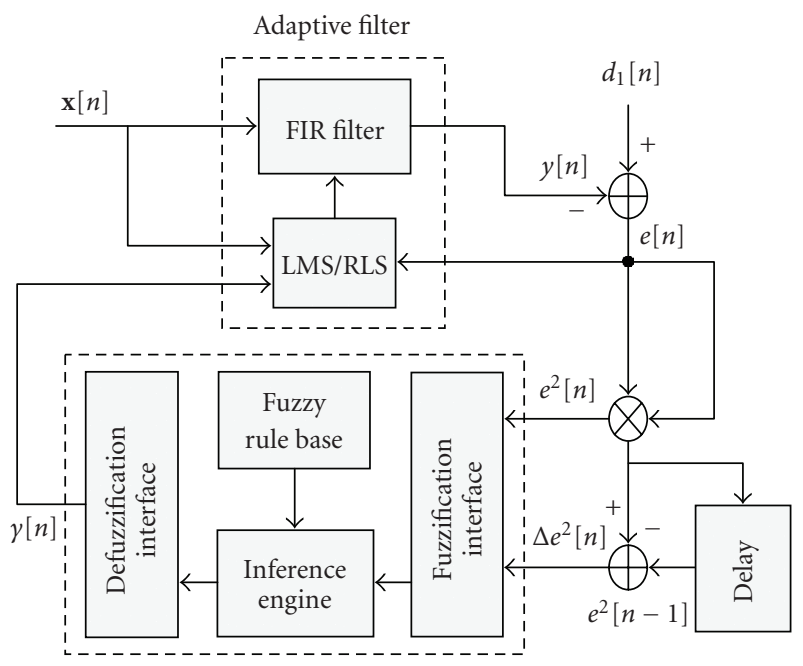

Fuzzy inference system (FIS)

FIGURE 1: Block diagram for the FLC-LMS and FLC-RLS algorithms.

The use of the exponential weighting factor $\lambda$ in the RLS algorithm, in general, is intended to ensure that the data in the distant past are "forgotten" in order to afford the possibility of following the statistical variations of the observable data when the filter operates in a nonstationary environment. To improve the dynamic-tracking capability of the adaptive filter, the RLS algorithm equipped with an adaptive iterative scheme is usually introduced for tuning the time-dependent value of $\lambda[n]$ at discrete time index $n$.

A novel approach, which uses the fuzzy inference system (FIS), is developed here to adjust adaptively the stepsize $\mu$ for the LMS algorithm or the forgetting factor $\lambda$ for the RLS algorithm at each time index. This proposed fuzzybased MMSE CDMA receiver provides superior convergence/tracking characteristic and smaller steady-state MSE over the conventional LMS and EW-RLS MMSE CDMA receivers. In what follows, the symbol $\gamma[n]$ is employed to stand for both time-dependent variables $\mu[n]$ and $\lambda[n]$ at time $n$.

In this paper, the two-input one-output FIS, which operates based on the principle of fuzzy logic proposed originally by Zadeh [22], takes in two inputs, the squared error $\left(e^{2}[n]\right)$, and the squared error variation $\left(\Delta e^{2}[n]\right)$ at the $n$th iteration. In general, the basic configuration of the FIS comprises four essential components, namely, (i) a fuzzification interface, (ii) a fuzzy rule base, (iii) an inference engine, and (iv) a defuzzification interface, which map two inputs $\left(e^{2}[n], \Delta e^{2}[n]\right)$ into an output $\gamma[n]$ for adaptive filtering schemes, as shown in Figure 1. The general format for the proposed FLC-LMS and FLC-RLS approaches to assign a suitable $\gamma[n]$ at time in$\operatorname{dex} n$ is formulated as

$$
\begin{gathered}
e[n]=d_{1}[n]-y[n]=d_{1}[n]-\mathbf{w}^{\dagger}[n] \mathbf{x}[n], \\
\Delta e^{2}[n]=\left|e^{2}[n]-e^{2}[n-1]\right|, \\
\text { FLC-LMS, FLC-RLS }: \gamma[n]=\operatorname{FIS}\left(e^{2}[n], \Delta e^{2}[n]\right),
\end{gathered}
$$


where $e[n], d_{1}[n]$, and $y[n]$ represent the error signal, the transmitted information bit, and the output of the adaptive filter, respectively, at the time instant $n$. The function of each component in the FIS is introduced briefly as follows.

\section{Fuzzification interface}

The fuzzification interface converts the values of each input parameter into suitable linguistic values that can be viewed as terms of fuzzy sets. These fuzzy sets are used for partitioning the continuous domains of the FIS input/output variables into a small number of $P$-overlapping regions labeled with linguistic terms, such as small (S), medium (M), large (L), and very large $(\mathrm{VL})$ in the case of $P=4$, as shown in Figures 2 and 3. In other words, the input variables to the FIS are transformed to the respective degrees to which they belong to each of the appropriate fuzzy sets by using membership functions (MBFs, possibility distributions, degrees of belonging). In this paper, the triangular-shaped MBF is employed and defined as follows:

$$
m_{B}(x)= \begin{cases}0, & \text { if } x<a, \\ \frac{x-a}{c-a}, & \text { if } x \in[a, c], \\ \frac{b-x}{b-c}, & \text { if } x \in[c, b], \\ 0, & \text { if } x>b,\end{cases}
$$

where $a, b$, and $c$ denote the lower bound, upper bound, and centroid of a triangle, respectively. Figures 2 and 3 illustrate three MBFs of (a) the squared error $\left(e^{2}\right)$, (b) the squared error variation $\left(\Delta e^{2}\right)$, and (c) the variable $\gamma$ for the FLC-LMS and FLC-RLS algorithms, respectively. In the case of $P=4$, four triangular MBFs with centroids of the very large $\left(\mathrm{VL}_{c}\right)$, large $\left(\mathrm{L}_{c}\right)$, medium $\left(\mathrm{M}_{c}\right)$, and small $\left(\mathrm{S}_{c}\right)$ MBFs, respectively, are selected to cover the entire universe of discourse (domain, universe), as illustrated in Figures 2 and 3. Thus, the FIS utilizes two fuzzy inputs, $\left(e^{2}[n], \Delta e^{2}[n]\right)$, and determines the respective degree to which they belong to each of the appropriate fuzzy sets via triangular MBFs. The crisp numerical inputs need to be limited to their respective domain of the input variables. The output of the fuzzification process demonstrates a fuzzy degree of membership between 0 and 1 .

\section{Fuzzy rule base}

The fuzzy rule base consists of the knowledge of the application domain and the attendant control goals. It consists of a fuzzy database and a linguistic (fuzzy) control rule base. The fuzzy database is used to define linguistic control rules and fuzzy data manipulation in the FLC. The control rule base characterizes the control goals and control policy by means of a set of linguistic control rules.

More generally, the operation of this component is to construct a set of fuzzy IF-THEN rules of the following form: for example, IF the squared error is "L" OR the squared error variation is "M," THEN the value of $\gamma$ is "M." The "OR"

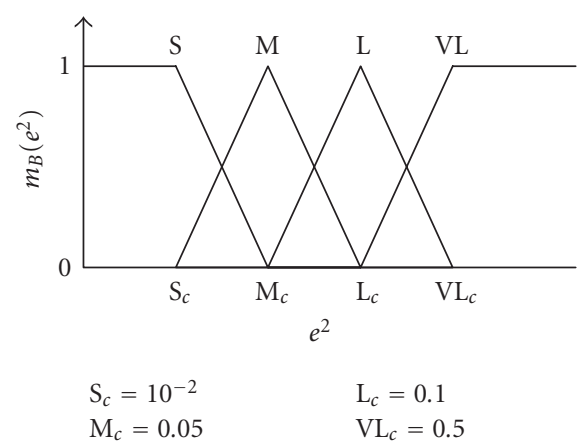

(a)

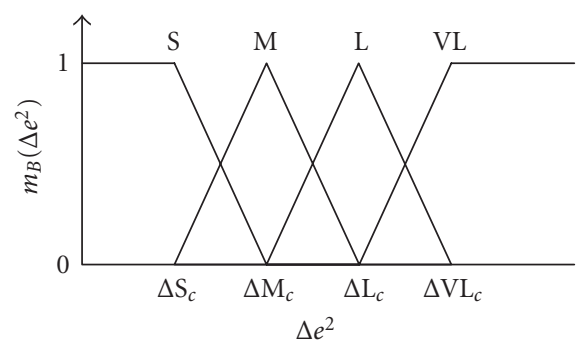

$$
\begin{array}{ll}
\Delta \mathrm{S}_{c}=10^{-3} & \Delta \mathrm{L}_{c}=0.1 \\
\Delta \mathrm{M}_{c}=10^{-2} & \Delta \mathrm{VL}_{c}=0.3
\end{array}
$$

(b)

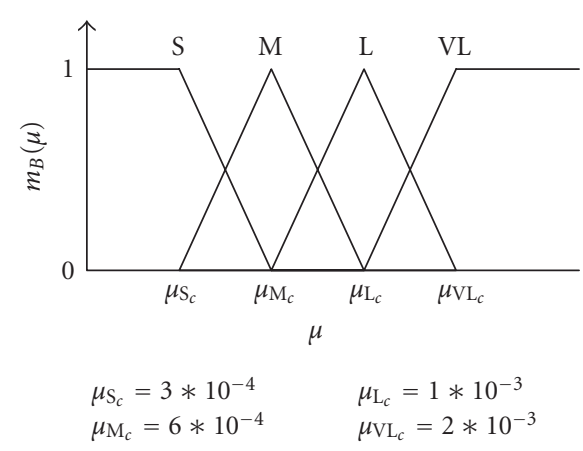

(c)

FIgure 2: Three MBFs of the FLC-LMS algorithm spread over their respective universe of discourse: (a) the squared error $e^{2}$, (b) the squared error variation $\Delta e^{2}$, and (c) the variable $\mu$.

operator, which combines the degrees of two input variables into a single value, selects the maximum value of the two. An important fact to note is that there exists no real causality between the antecedent (IF-part) and the consequent (THEN-part) in Boolean logic. This fact shows a big difference in human reasoning. Hence, the set of fuzzy IF-THEN rules expresses cause-effect relations, and fuzzy logic is used as a tool for transferring such structured human knowledge into feasible algorithms. Specifically, these IF-THEN fuzzy rules have been derived from the usual rule of thumb for the purpose of adjusting the value of $\gamma$. The relations between the MBFs and the fuzzy rules in the FIS of the LMS and RLS algorithms are illustrated in Figures 4 and 5. 


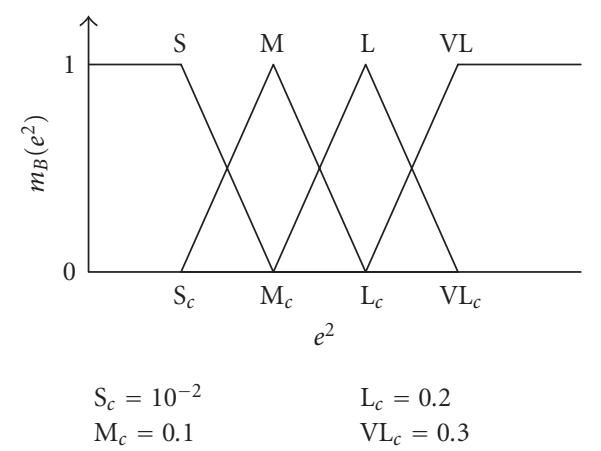

(a)

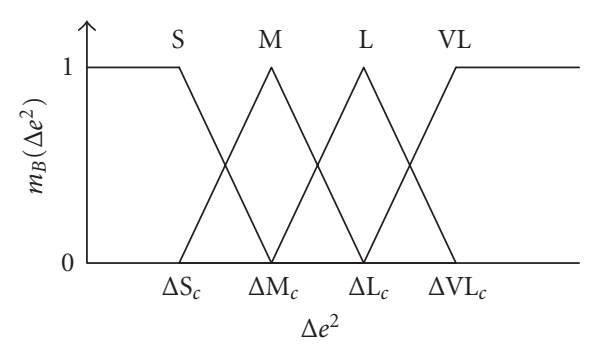

$$
\begin{array}{ll}
\Delta \mathrm{S}_{c}=5 * 10^{-3} & \Delta \mathrm{L}_{c}=0.1 \\
\Delta \mathrm{M}_{c}=5 * 10^{-2} & \Delta \mathrm{VL}_{c}=0.2
\end{array}
$$

(b)

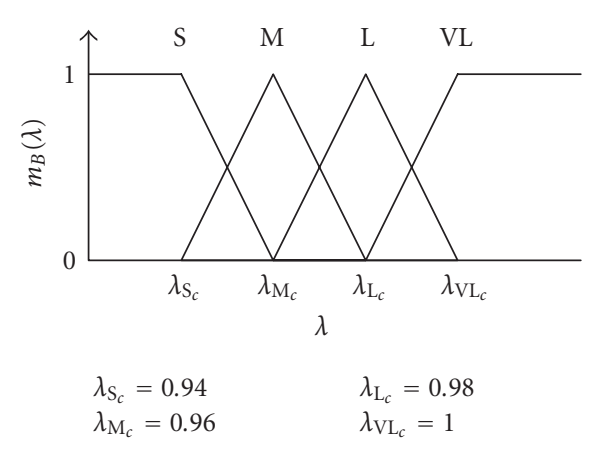

(c)

FIgURE 3: Three MBFs of the FLC-RLS algorithm spread over their respective universe of discourse: (a) the squared error $e^{2}$, (b) the squared error variation $\Delta e^{2}$, and (c) the variable $\lambda$.

In this paper, we claim that the convergence is just at the beginning in case of a "VL" $e^{2}$ and a "VL" $\Delta e^{2}$ and a very large step size is used to increase its convergence rate. On the other hand, the adaptive filter is assumed to operate in the steadystate status when both $e^{2}$ and $\Delta e^{2}$ show "S" and a small step size is adopted to lower its steady-state MSE. In particular, we may declare that a huge estimation error has occurred when $e^{2}$ is " $\mathrm{S}$ " and $\Delta e^{2}$ indicates "VL" and a small step size is assigned to system in order to stabilize system performance. This particular rule prevents algorithms from overreacting to some abnormal conditions which cause an unexpectedly abrupt jump in the error, therefore, making them robust

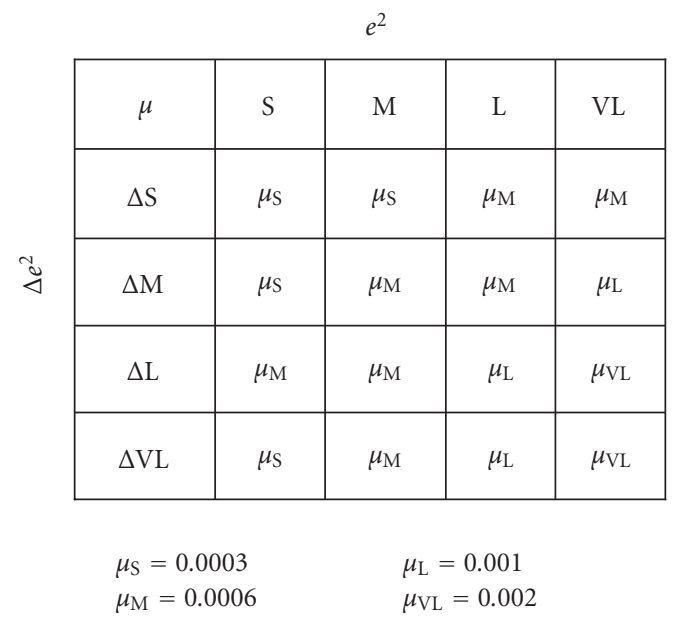

FIGURE 4: Predicate box for the FLC-LMS algorithm.

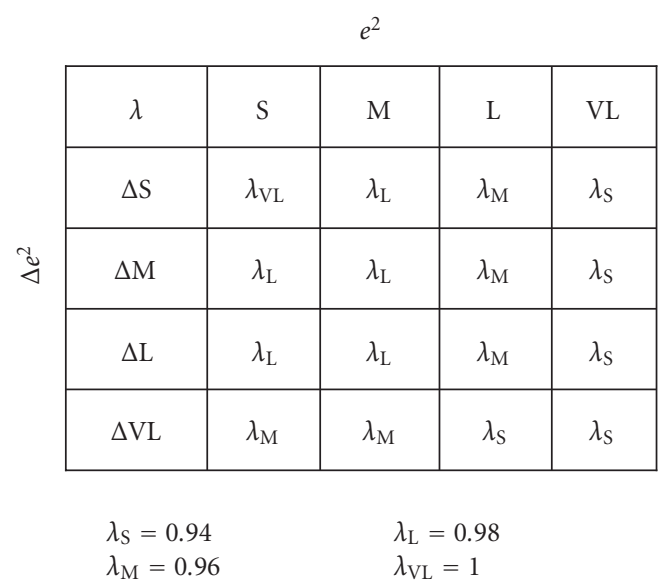

FIgURE 5: Predicate box for the FLC-RLS algorithm.

algorithms while compared to the other numerical-based algorithms. The key concepts of the fuzzy rules are shared and used to establish a common foundation for both the LMS and RLS algorithms in order to make the best choice for the $\gamma$. All the fuzzy inference rules used for the proposed LMS and RLS algorithms are summarized in Figures 4 and 5, respectively.

\section{Inference engine}

The inference engine in Figure 1 is a decision-making logic mechanism of the FIS. The fuzzified input variables, which contain the degrees of the antecedents (IF-part) of a fuzzy rule, need to be combined using a fuzzy operator to obtain a single value. Two built-in fuzzy operators of the "OR" and "AND," which select, respectively, the "maximum" and "minimum" of the two values, are chosen mostly to implement combinations in the FIS. We have examined these two commonly used fuzzy operators, "AND" and "OR," as 


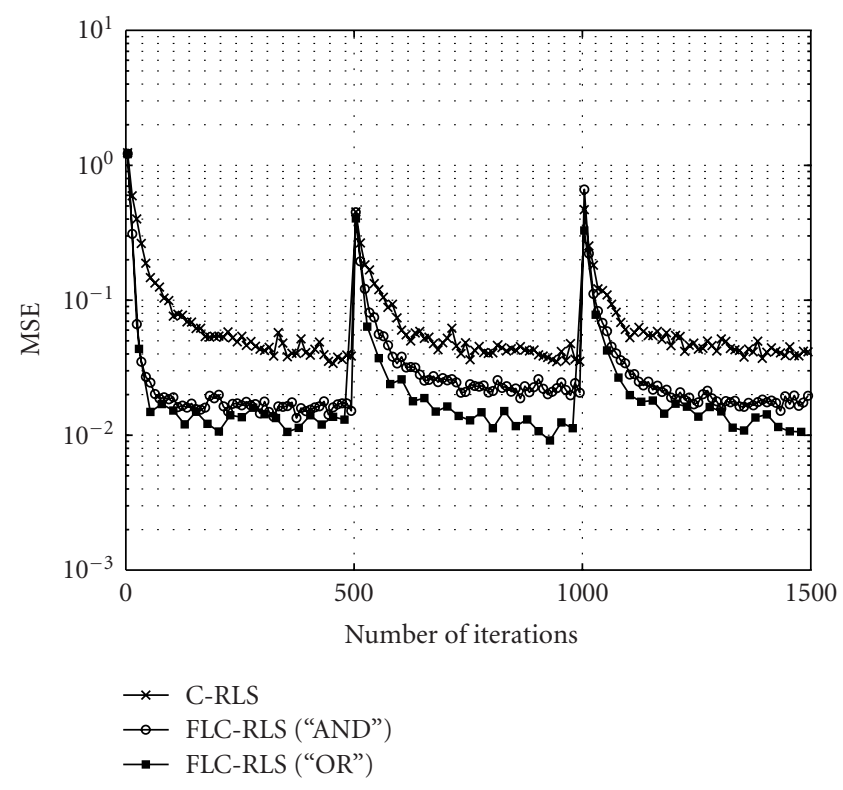

Figure 6: Mean square error (MSE) versus the number of iterations $L$ parameterized by fuzzy operators for the FLC-RLS with the parameters $K=10\left(K_{\text {intra }}=8, K_{\text {inter }}=2\right), K_{l}=3, M=1$, SNR = $20 \mathrm{~dB}$, and a multipath fading rate $=1 / 500$ fade cycle/symbol.

shown in Figure 6. In general, the use of the "OR" operator is able to produce better performance than the "AND" operator in multipath Rayleigh-fading channels. Subsequently, this is followed by the implication process, which defines the reshaping task of the consequent (THEN-part) of the fuzzy rule based on the antecedent. The input for the implication process is a single number given by the antecedent, and the output is a fuzzy set. Implication process is implemented for each rule. A min (minimum) operation is generally employed to truncate the output fuzzy set for each rule. Since decisions are based on the testing of all of the rules in an FIS, the rules need to be combined in some manner in order to make a decision. Aggregation is the process by which the fuzzy sets that represent the outputs of each rule are combined into a single fuzzy set. Aggregation only occurs once for each output variable, just prior to the process of defuzzification. The input of the aggregation process is the list of truncated output functions returned by the implication process for each rule. The output of the aggregation process is one fuzzy set for each output variable.

\section{Defuzzification interface}

Before feeding the signal to the adaptive filter, we need a defuzzification process to get a crisp decision. The procedure for obtaining a crisp output value from the resulting fuzzy set is called defuzzification. Note the subtle difference between fuzzification and defuzzification: fuzzification represents the transformation of a crisp input into a vector of membership degrees, and defuzzification transforms a fuzzy set into a crisp value. In other words, the defuzzification interface converts fuzzy control decision into crisp, nonfuzzy (physical) control signals. These control signals are applied to adjust the value of the variable $\gamma$ in order to improve convergence/tracking capability of the proposed CDMA receiver. The crisp nonfuzzy control command is computed by the centroid-defuzzification method. The reason for using the center-of-gravity or fuzzy centroid-defuzzification method instead of other defuzzification methods such as first-, middle-, and largest-of-maximum and center-of-area for singletons is because the fuzzy centroid-defuzzification method yields an excellent performance, for example, the smallest MSE, and grants itself well to be implemented on the DSP. The other approaches require comparison operations to be carried out which complicate the implementation of defuzzification in DSP. The centroid-defuzzification output $\gamma[n]$ is calculated by [23]

$$
\gamma[n]=\frac{\sum_{i=1}^{q} \gamma_{i}[n] m_{B}\left(\gamma_{i}[n]\right)}{\sum_{i=1}^{q} m_{B}\left(\gamma_{i}[n]\right)},
$$

where $q$ is the number of discrete samples of the output MBF, $\gamma_{i}[n]$ is the value at the location used in approximating the area under the aggregated MBF, and $m_{B}\left(\gamma_{i}[n]\right) \in[0,1]$ indicates the MBF value at location $\gamma_{i}[n]$. To alleviate the computational load in the centroid-defuzzification calculation, fewer points $q$ must be used. The calculation of $\gamma[n]$ in (26) returns the center of the area under the aggregated MBFs. The adaptive parameter $\gamma[n]$ which is determined from (26) is used to update the adaptive filter coefficients in (16) and (21) of Section 3.1.

\subsection{Computational complexity analysis}

We first evaluate the extra complexity requirements by introducing the (2-to-1)-FIS in the adjustment of value $\gamma$. In general, the increase in complexity comes in the form of special instructions, to perform table lookups and comparisons in the IF-THEN rules and additional multiplications and additions in the defuzzification process. Table 1 lists the required multiplications, additions, and special instructions to perform the FIS, which come primarily from the preparation and fuzzification of two input variables, fuzzy OR operations, fuzzy minimum implication, aggregation of the output, and the centroid-defuzzification output process $[24,25]$.

For simplicity of notation, let $\Upsilon$ stand for the number of $\left(N+K_{l}-1\right)$ in what follows. The computational complexity of the conventional adaptive LMS algorithm, in terms of multiplications and additions, can be easily shown to be equal to $2 M \Upsilon+1$ and $2 M \Upsilon+1$ per tap-weight update, respectively. In [12], Harris et al. proposed the VSS-LMS approach which requires $6 M Y$ multiplications, $2 M \Upsilon$ additions, $\Upsilon$ sign operations, and $2 \Upsilon$ compares per iteration. The VSSLMS algorithm proposed in [13] by Kwong and Johnston needs $2 M \Upsilon+4$ multiplications, $2 M Y+2$ additions, and 2 compares. The complexity cost of the proposed FLC-LMS is $2 M \Upsilon+q+3$ multiplications, $2 M \Upsilon+2 q+2$ additions, and extra special instructions (i.e., a total of 24 lookups +16 compares $+16 q$ max. operations.) per iteration. Thus, the load of 
TABLE 1: Computational load (per iteration) for the FIS.

\begin{tabular}{l|ccc}
\hline FIS & Mult. and div. & Add. and sub. & Special instructions \\
\hline Two fuzzified inputs & 1 for computing $e^{2}$ & 1 for computing $\Delta e^{2}$ & 8 lookups \\
Fuzzy OR operator & - & - & 16 compares \\
Fuzzy min implication & - & - & 16 lookups \\
Aggregation of output & - & $2 q$ & $16 q$ max. operations \\
using max operator & $q+1$ & $2 q+1$ & - \\
$\begin{array}{l}\text { Defuzzification using centroid } \\
\text { method over q-point interval }\end{array}$ & $q+2$ & 24 lookups +16 compares \\
(2-to-1)-FIS & & & $+16 q$ max. operations \\
\hline
\end{tabular}

TABLE 2: Computational complexity (per iteration) for the LMS, RLS, FLC-LMS, and FLC-RLS.

\begin{tabular}{l|ccc}
\hline Algorithm & Mult. and div. & Add. and sub. & Special instructions \\
\hline C-LMS & $2 M \Upsilon+1$ & $2 M \Upsilon+1$ & - \\
Harris's VSS-LMS [12] & $6 M \Upsilon$ & $2 M \Upsilon$ & $\Upsilon$ sign operations + 2צ compares \\
Kwong's VSS-LMS [13] & $2 M \Upsilon+4$ & $2 M \Upsilon+2$ & 2 compares \\
FLC-LMS & $2 M \Upsilon+q+3$ & $2 M \Upsilon+2 q+2$ & 24 lookups + 16 compares \\
C-RLS & $2(M \Upsilon)^{2}+4 M \Upsilon$ & $2(M Y)^{2}+3 M \Upsilon+2$ & $+16 q$ max. operations \\
FLC-RLS & $2(M \Upsilon)^{2}+4 M \Upsilon+q+2$ & $2(M \Upsilon)^{2}+3 M \Upsilon+2 q+3$ & 24 lookups + 16 compares \\
& & & $+16 q$ max. operations \\
\hline
\end{tabular}

the FLC-LMS is slightly heavier than that of the conventional LMS (C-LMS), but it is still a tolerable level.

The conventional RLS (C-RLS) algorithm requires $2(M \Upsilon)^{2}+4 M \Upsilon$ multiplications and $2(M \Upsilon)^{2}+3 M \Upsilon+2$ additions, which involve the derivations of the filtered information vector $\mathbf{v}[n] \triangleq \mathbf{P}[n-1] \mathbf{x}[n]$, gain vector $\mathbf{k}[n]$, a priori estimation error $\xi[n]$, weight vector $\mathbf{w}[n]$, and autocorrelation inverse $\mathbf{P}[n]$. It is evident that the C-RLS approach based on the matrix inversion lemma for recursively updating $\mathbf{R}_{\mathbf{x}}^{-1}[n]$ requires $\mathcal{O}\left((M \Upsilon)^{2}\right)$ complexity. It should be emphasized that the proposed FLC-RLS is able to achieve the same order of complexity as the conventional one, but produces a better performance in convergence and data demodulation. Finally, the computational complexity, in terms of multiplications, additions, and special instructions, of the compared algorithms is summarized in Table 2 .

\section{REVIEW OF EXISTING LMS AND RLS ALGORITHMS}

In this section, three variable step-size LMS (VSS-LMS) approaches (Algorithms I III) and three variable forgetting factor (VFF-RLS) RLS approaches (Algorithms IV VI), which we use to analyze and compare the behavior of the proposed FLC-LMS and FLC-RLS algorithms in the simulations, are explained briefly.

\section{Algorithm I (Harris's VSS-LMS)}

In order to improve the performance of the LMS algorithm, the class of VSS-LMS algorithms was introduced. The
VSS-LMS algorithm proposed in [12] by Harris et al. controls the step size by examining the polarity of successive samples of the estimation errors. If there are $m_{0}$ consecutive sign changes (i.e., in steady-state mode), the step size is decreased by an appropriate amount, whereas if there are $m_{1}$ consecutive signs unchanged (i.e., in tracking mode), the step size is increased by an appropriate amount [12]. The thresholds of $m_{0}$ and $m_{1}$ are selected based on the requirements and applications.

\section{Algorithm II (Kwong's VSS-LMS)}

Kwong and Johnston [13] proposed an alternative scheme that adjusts the step size based on the fluctuation of the prediction squared error. The algorithm in [13] uses a timevariable step size, which is adjusted as follows:

$$
\mu^{\prime}[n+1]=f(\mu[n], e[n])=\kappa_{1} \mu[n]+\kappa_{2} e^{2}[n],
$$

where $\kappa_{1}$ and $\kappa_{2}$ are two positive scalars, $e[n]$ is the filter output error at time instant $n, f(\cdot)$ denotes the function of the arguments, and

$$
\mu[n+1]= \begin{cases}\mu_{\max }, & \text { if } \mu^{\prime}[n+1]>\mu_{\max }, \\ \mu_{\min }, & \text { if } \mu^{\prime}[n+1]<\mu_{\min } \\ \mu^{\prime}[n+1], & \text { otherwise. }\end{cases}
$$

Here $\mu_{\max }$ and $\mu_{\min }$ are the minimum and the maximum values allowed for the step size $\left(0<\mu_{\min }<\mu_{\max }\right)$, respectively. 
The constant $\mu_{\max }$ is chosen to ensure that the MSE of the algorithm remains bounded. The value of $\kappa_{1}$ needs to be selected in the range of $(0,1)$ to provide exponential forgetting.

\section{Algorithm III (Aboulnasr's VSS-LMS)}

In [13], the transient and steady-state analysis of the VSSLMS is given and the theoretical misadjustment is derived for both stationary and nonstationary cases. However, from the analysis presented in [13] the value of the misadjustment and the convergence speed depend on both coefficients $\kappa_{1}$ and $\kappa_{2}$. Therefore, we can conclude that the VSS-LMS increases the convergence speed but still has the drawback between a fast convergence and a small steady-state error. Another adaptive LMS algorithm with a time-varying step size was introduced by Aboulnasr and Mayyas in [26] to improve the steady-state performance of the VSS-LMS algorithm in [13]. The stepsize update of the VSS-LMS algorithm of [26] is described by the following equations:

$$
\begin{aligned}
\mu^{\prime}[n+1] & =f(\mu[n], p[n])=\kappa_{1} \mu[n]+\kappa_{2} p^{2}[n] \\
\mu[n+1] & = \begin{cases}\mu_{\max }, & \text { if } \mu^{\prime}[n+1]>\mu_{\max } \\
\mu_{\min }, & \text { if } \mu^{\prime}[n+1]<\mu_{\min } \\
\mu^{\prime}[n+1], & \text { otherwise }\end{cases}
\end{aligned}
$$

where

$$
p[n]=\kappa_{3} p[n-1]+\left(1-\kappa_{3}\right) e[n] e[n-1] .
$$

Constants $\kappa_{1}$ and $\kappa_{2}$ are the same as those of Kwong's VSSLMS algorithm. The positive constant $\kappa_{3}$ is an exponential weighting parameter. Using an approximation of the error autocorrelation $p[n]$ in the step-size update, the influence of the measurement noise is reduced and the algorithm performs better at the steady state. However, also in the case of this algorithm the steady-state misadjustment depends on all three parameters $\left(\kappa_{1}, \kappa_{2}\right.$, and $\left.\kappa_{3}\right)$, so the dependence between the convergence speed and the steady-state error still exists.

\section{Algorithm IV (the EW-RLS with an optimal fixed forgetting factor)}

In [27], an explicit expression of the optimal forgetting factor for the EW-RLS algorithms (OFFF-RLS) is derived based on a prior Doppler power spectrum of the Jakes' fading channel model [28] as follows:

$$
\lambda_{\mathrm{opt}}=1-\left(\frac{8 \pi^{2} f_{D}^{2} E_{\mathbf{x}}}{K_{l} \sigma_{n}^{2}}\right)^{1 / 3}
$$

where $E_{\mathbf{X}}$ is the average energy of $\mathbf{x}[n]$. It is reflected in (31) that $\lambda_{\text {opt }}$ needs to be updated by $f_{D}$ and SNR.

\section{Algorithm V (the gradient-based variable forgetting factor $R L S$ )}

The control of the forgetting factor is to adjust $\lambda$ to minimize the error criterion, given as

$$
J[n]=\frac{1}{2} E\left\{|\xi[n]|^{2}\right\}
$$

The essence of the gradient-based variable forgetting factor RLS (GVFF-RLS) algorithm [29] is to use the dynamic equation of the MSE to calculate the gradient recursively rather than using the noisy instantaneous estimate. By using the steepest descent (SD) method, the forgetting factor is updated recursively as

$$
\lambda[n]=\left[\lambda[n-1]-\alpha \cdot \nabla_{\lambda}(J[n])\right]_{\lambda_{-}}^{\lambda_{+}}
$$

where $\nabla_{\lambda}(\cdot) \triangleq \partial(\cdot) / \partial \lambda$ and $\alpha$ is a positive small learning-rate parameter. The bracket in (33) is a clipper function with the ceiling $\lambda_{+}$and the floor $\lambda_{-}$. Thus, taking the derivative of $J[n]$ in (32) with respect to $\lambda$, the minimization problem of (32) yields a set of iterative equations as follows:

$$
\begin{gathered}
\mathbf{k}[n]=\frac{\mathbf{P}[n-1] \mathbf{x}[n]}{\lambda[n-1]+\mathbf{x}^{H}[n] \mathbf{P}[n-1] \mathbf{x}[n]}, \\
\xi[n]=d_{1}[n]-\mathbf{w}^{H}[n-1] \mathbf{x}[n], \\
\mathbf{w}[n]=\mathbf{w}[n-1]+\xi^{*}[n] \mathbf{k}[n], \\
\mathbf{P}[n]=\lambda^{-1}[n-1]\left(\mathbf{I}-\mathbf{k}[n] \mathbf{x}^{H}[n]\right) \mathbf{P}[n-1], \\
\lambda[n]=\left[\lambda[n-1]+\alpha \cdot \operatorname{Re}\left\{\mathbf{\Phi}^{H}[n-1] \mathbf{x}[n] \xi^{*}[n]\right\}\right]_{\lambda_{-}}^{\lambda_{+}}, \\
\mathbf{S}[n]=\nabla_{\lambda}(\mathbf{P}[n]) \\
=\lambda^{-1}[n]\left\{\left(\mathbf{I}-\mathbf{k}[n] \mathbf{x}^{H}[n]\right) \mathbf{S}[n-1]\right. \\
\left.\times\left(\mathbf{I}-\mathbf{x}[n] \mathbf{k}^{H}[n]\right)+\mathbf{x}[n] \mathbf{k}^{H}[n]-\mathbf{P}[n]\right\} \\
\mathbf{\Phi}[n]=\nabla_{\lambda}(\mathbf{w}[n])=\left(\mathbf{I}-\mathbf{k}[n] \mathbf{x}^{H}[n]\right) \mathbf{\Phi}[n-1]+\mathbf{S}[n] \mathbf{x}[n] \xi^{*}[n] .
\end{gathered}
$$

\section{Algorithm VI (VFF-LLS algorithm)}

In [30], the cost function with the use of noise variance weighting is adopted for better performance, which is defined as

$$
J^{\prime}[n]=\frac{1}{2} E\left\{\frac{|\xi[n]|^{2}}{\sigma_{n}^{2}}\right\}
$$

The optimal vector of $\mathbf{w}[n]$ at time $n$ is therefore calculated by the minimization of the $J^{\prime}[n]$ in (41). In other words, differentiating $J^{\prime}[n]$ with respect to $\lambda$, the minimization 
problem of (41) leads to the following:

$$
\begin{gathered}
\mathbf{k}[n]=\frac{\mathbf{P}[n] \mathbf{x}[n]}{\sigma_{n}^{2}}, \\
\lambda[n]=\left[\lambda[n-1]+\frac{\alpha}{\sigma_{n}^{2}} \cdot \operatorname{Re}\left\{\boldsymbol{\Phi}^{H}[n-1] \mathbf{x}[n] \xi^{*}[n]\right\}\right]_{\lambda_{-}}^{\lambda_{+}}, \\
\mathbf{S}[n]=\lambda^{-1}[n]\left\{\left(\mathbf{I}-\mathbf{k}[n] \mathbf{x}^{H}[n]\right) \mathbf{S}[n-1]\right. \\
\left.\left(\mathbf{I}-\mathbf{x}[n] \mathbf{k}^{H}[n]\right)+\frac{\mathbf{x}[n] \mathbf{k}^{H}[n]}{\sigma_{n}^{2}}-\mathbf{P}[n]\right\}, \\
\mathbf{\Phi}[n]=\left(\mathbf{I}-\mathbf{k}[n] \mathbf{x}^{H}[n]\right) \mathbf{\Phi}[n-1]+\mathbf{S}[n] \mathbf{x}[n] \frac{\xi^{*}[n]}{\sigma_{n}^{2}} .
\end{gathered}
$$

The equations for $\xi[n], \mathbf{w}[n]$, and $\mathbf{P}[n]$ remain unchanged (i.e., the same expressions as (35)-(37)).

\section{NUMERICAL RESULTS}

In computer simulations, an asynchronous BPSK DS-CDMA system with the number of users $K=K_{\text {intra }}+K_{\text {inter }}$ is considered in the presence of frequency-selective multipath fading. Users $1 \sim K_{\text {intra }}$ are assumed to be users in the active cell and users $1 \sim K_{\text {inter }}$ are the intercell interferers. The spreading sequence of each user in the active cell is a Gold sequence of length $N=31$ while the spreading codes for intercell interferers are random codes. For a frequency-selective multipath fading channel, each user signal is assumed to experience $K_{l}=3$ independent Rayleigh-fading paths due to multipath reflections and independent angle of arrival (AOA) distributed uniformly in $(-\pi / 2, \pi / 2)$. The relative delays of different users and paths are multiples of $T_{c}$. The user of interest, say of user 1, is the user to be acquired in the presence of intersymbol interference (ISI) and multiple-access interference (MAI). The information about the in-cell and inter-cell interferers is assumed to be unavailable at the base station receiver. The antenna array receiver employs uniformly spaced linear-array antenna with $M$ elements of halfwavelength spacing. In addition, the linear MMSE receiver is assumed to employ a known training sequence $\left\{d_{1}[k]\right\}_{k=1}^{N_{T}}$ of length $N_{T}=100$, followed by a DD adaptation for rapid adaptive convergence. The settings of the MBFs parameters required for the FIS of the FLC-LMS and FLC-RLS are shown in Figures 2 and 3, respectively. It must be emphasized that these are not the only settings for the input and output fuzzy variables. In simulations, the performance of the proposed FLC-based algorithms is evaluated and compared with three VSS-LMS (i.e., Harris's VSS-LMS [12] with $m_{0}=m_{1}=4$, Kwong's VSS-LMS [13] with $\kappa_{1}=0.97$ and $\kappa_{2}=4.8 \times 10^{-4}$, and Aboulnasr's VSS-LMS [26] with $\kappa_{1}=0.97, \kappa_{2}=4.8 \times 10^{-4}$, and $\left.\kappa_{3}=0.1\right)$ and three VFFRLS (i.e., the OFFF-RLS [27], GVFF-RLS [29], and VFF-LLS [30]) algorithms. The LMS step size $\mu$ is chosen to be conservatively limited by $2 / \operatorname{trace}\left(\mathbf{R}_{\mathbf{X}}[n]\right)$, where trace $\left(\mathbf{R}_{\mathbf{X}}[n]\right)$ indicates the total tap-input power of the filter. With this step size, the LMS filter, given the limitations of the independence assumption [21], guarantees the convergence to the optimal Wiener solution in the mean and in the mean square. The
RLS forgetting factor $\lambda$ is assumed to be bounded by the values of $0.94\left(\lambda_{-}\right)$and $1.0\left(\lambda_{+}\right)$in simulations. The learningrate parameter $\alpha$ is set to 0.005 . All experimental curves are obtained using $2 \times 10^{4}$ independent trials.

First of all, the convergence behavior and the steady-state performance of the proposed FLC-LMS and FLC-RLS algorithms is presented in Figure 7 in terms of the number of iterations $L$ in the presence of the Rayleigh-fading channel with a medium-fading rate $=1 / 500$ fade cycle/symbol. Figures 7 (a) and 7 (b) presents the results with the parameters $K=10$ $\left(K_{\text {intra }}=8, K_{\text {inter }}=2\right), K_{l}=3, M=1$, and SNR $=10 \mathrm{~dB}$. In general, a large step size causes a faster convergence speed and a larger MSE fluctuation. The characteristic exhibits entirely reverse when a small step size is utilized. Experimental results in Figure 7(a) show that the proposed FLC-LMS approach possesses a faster rate of convergence without trading off the steady-state performance. Thus, it is evident that the behavior of the FLC-LMS in convergence and steady state takes the advantage of both the large and small step sizes. Moreover, the FLC-LMS algorithm produces a comparable convergence and steady-state performance to the C-RLS algorithm. Furthermore, it is demonstrated in Figure 7(b) that the FLC-RLS algorithm provides the fastest speed of convergence and the smallest steady-state misadjustment among all the other algorithms. The proposed FLC-RLS algorithm possesses a superior capability of transient response and dynamic tracking to a sudden environment change that makes it possible to operate in a dynamic fading channel. The comparison between the FLC-LMS and FLC-RLS algorithms is provided as well and shown in Figure 7. The FLC-RLS algorithm produces a better convergence characteristic and a lower steady-state MSE as compared to the FLC-LMS algorithm, but incurs a heavier computation and implementation load.

Figure 8 demonstrates the bit-error-rate (BER) performance of the LMS-based, RLS-based, FLC-LMS, and FLC-RLS approaches as a function of SNR under the MAI and Rayleigh-fading environment. Evidently, the FLCRLS achieves a much better BER performance than other schemes, because of the use of a fuzzy variable forgetting factor $\lambda$ in response to the time-varying channels. Simulation results in Figure 8 show that a significant improvement in BER performance is achieved by the FLC-based algorithms. More specifically, the FLC-LMS approach in Figure 8(a) achieves, respectively, $3 \mathrm{~dB}$ and $6 \mathrm{~dB}$ over the Kwong's and Aboulnasr's VSS-LMS algorithms at a specific BER requirement of 0.25 (marked by a dashed line in Figure 8(a)) for multipath Rayleigh-fading channels. The BER improvement is enhanced further when the FLC-LMS algorithm is compared with the C-LMS and Harris's VSS-LMS algorithms. Moreover, it is clearly seen from Figure 8(b) that the FLCRLS approach accomplishes $8 \mathrm{~dB}, 4 \mathrm{~dB}, 2.1 \mathrm{~dB}$, and $1.5 \mathrm{~dB}$ over the C-RLS, OFFF-RLS, GVFF-RLS, and VFF-RLS, respectively, at a fixed BER of $4 \times 10^{-2}$ (marked by a dashed line in Figure 8(b)). Also, results show that the FLC-LMS does not perform as good as the RLS-based approaches in data demodulation, due to its slow convergence speed, but the FLC-LMS provides a much simpler implementation. 


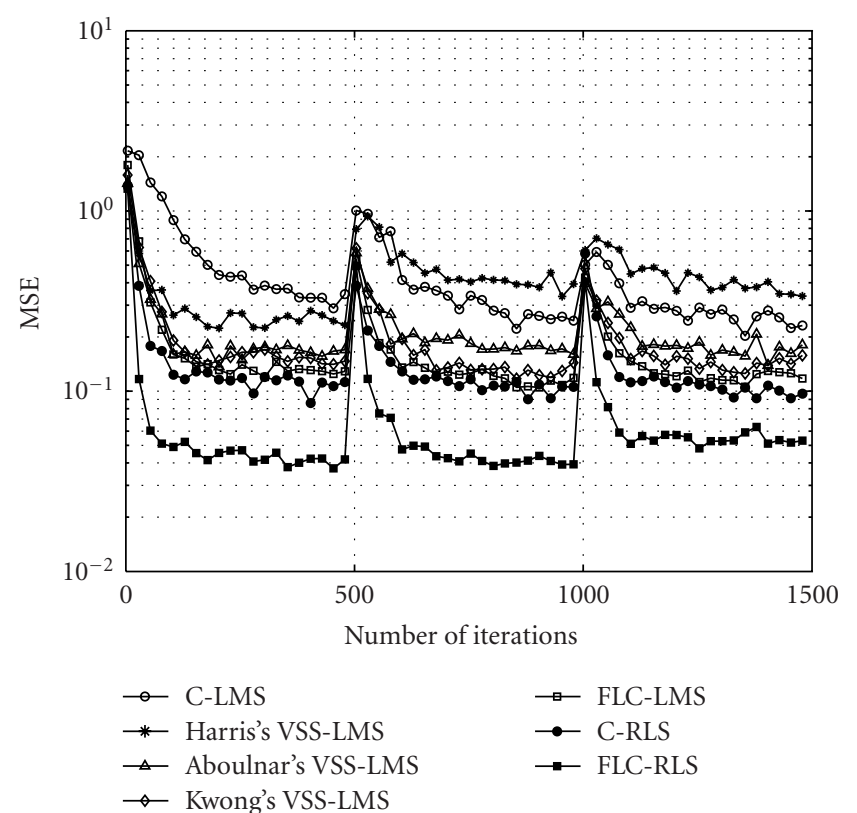

(a)

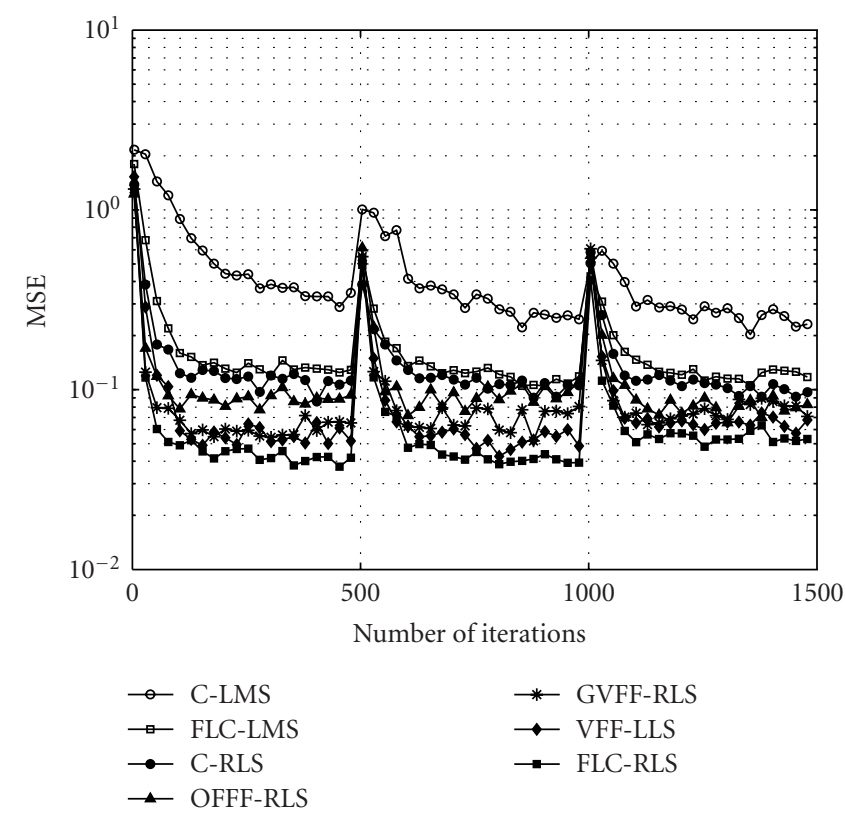

(b)

Figure 7: Mean square error versus the number of iterations $L$ for (a) C-LMS, VSS-LMS, and FLC-LMS, and (b) C-RLS, VFF-RLS, and FLC-RLS, both cases with the parameters $K=10\left(K_{\text {intra }}=8\right.$, $\left.K_{\text {inter }}=2\right), K_{l}=3, M=1, \mathrm{SNR}=10 \mathrm{~dB}$, and a multipath fading rate $=1 / 500$ fade cycle/symbol.

In Figure 9, the BER performance for various LMS and RLS algorithms is presented in terms of the number of users $K$ for $K_{l}=3, M=2, \mathrm{SNR}=10 \mathrm{~dB}$, and a multipath fading rate $=1 / 500$ fade cycle/symbol. Each interfering user in the simulations is assumed to have a received power equal to the desired user. It should be pointed out that an increase

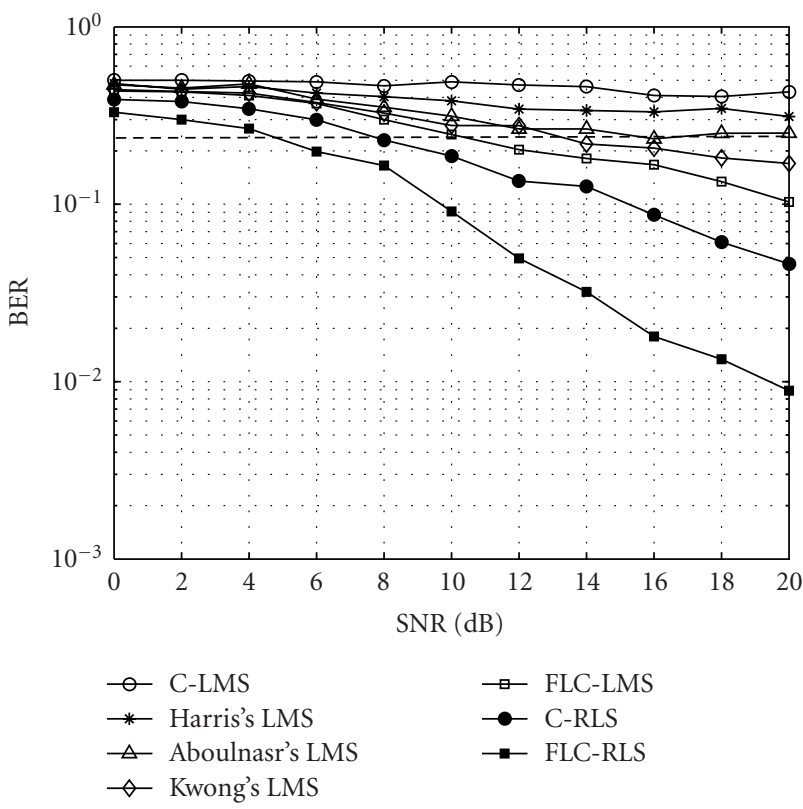

(a)

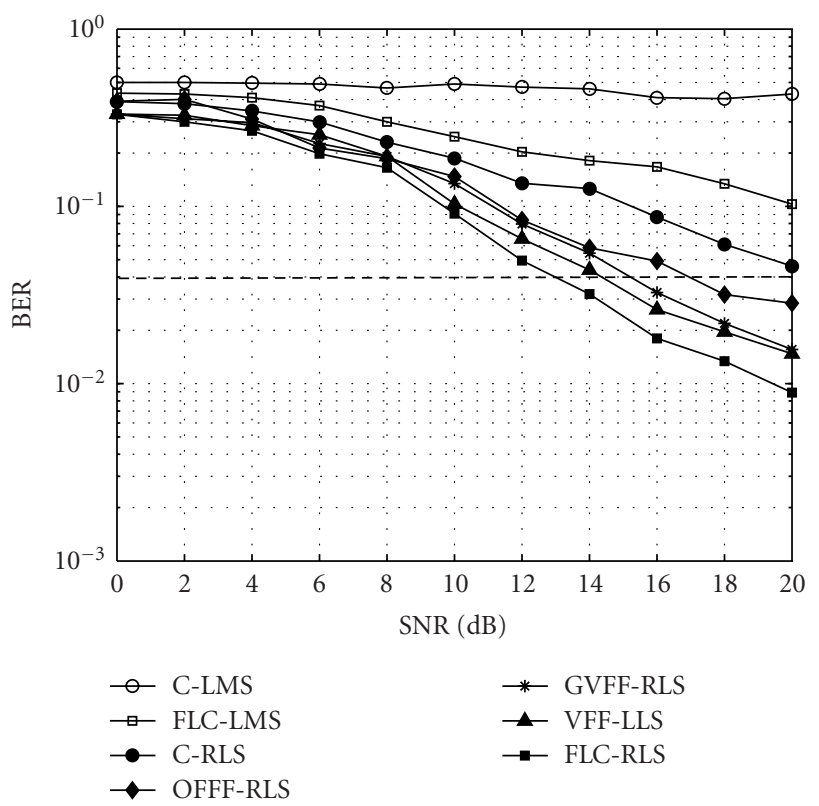

(b)

FIGURE 8: BER performance versus SNR for (a) C-LMS, VSS-LMS, and FLC-LMS, and (b) C-RLS, VFF-RLS, and FLC-RLS, both cases with the parameters $K=17\left(K_{\text {intra }}=15, K_{\text {inter }}=2\right), K_{l}=3, M=2$, and a multipath fading rate $=1 / 500$ fade cycle/symbol.

in system capacity is achieved under a fixed performance requirement when either the FLC-LMS or the FLC-RLS algorithm is employed.

Figure 10(a) depicts the BER performance of the proposed FLC-LMS and FLC-RLS algorithms as a function of SNR parameterized by the size of the FIS and Figure 10(b) 


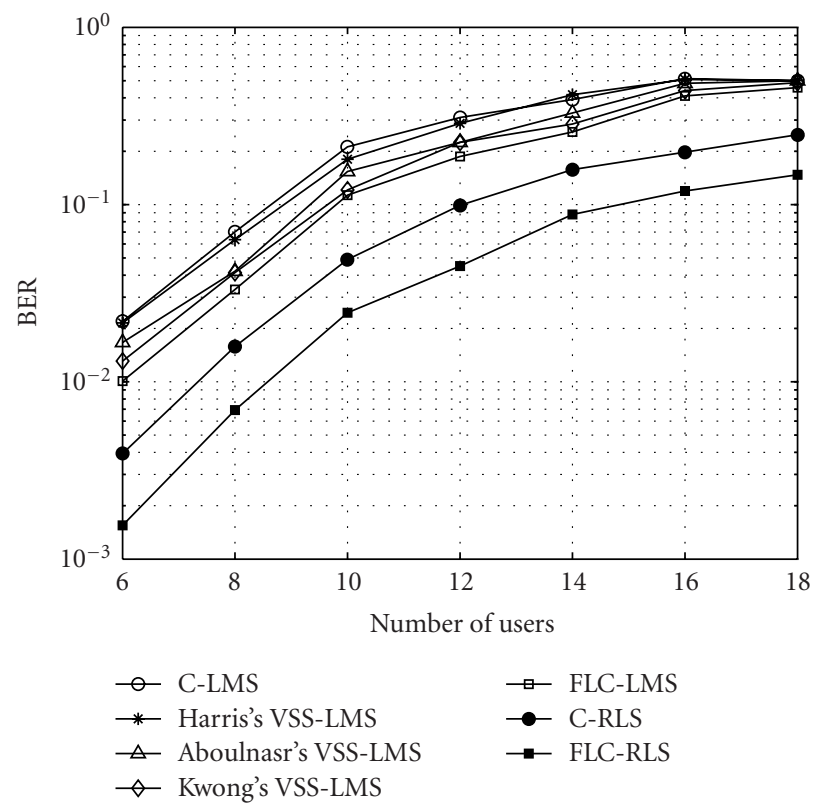

(a)

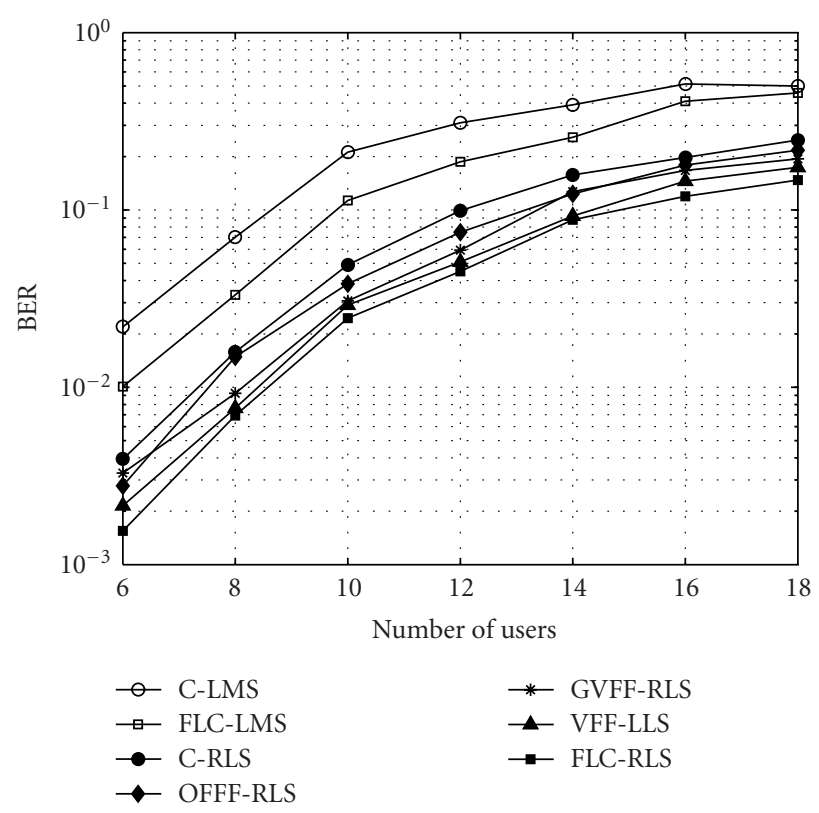

(b)

FIGURE 9: BER performance versus the number of users $K$ for (a) C-LMS, VSS-LMS, and FLC-LMS, and (b) C-RLS, VFF-RLS, and FLC-RLS, both cases with the parameters $K_{l}=3, M=2$, SNR = $10 \mathrm{~dB}$, and a multipath fading rate $=1 / 500$ fade cycle/symbol.

illustrates the BER performance of the FLC-RLS algorithm versus SNR parameterized by the $P$-partitioned MBFs for $K=17\left(K_{\text {intra }}=15, K_{\text {inter }}=2\right), K_{l}=3, M=2$, and a multipath fading rate $=1 / 500$ fade cycle/symbol. Experimental results in Figure 10(a) show that the DS-CDMA systems with a two-input $\left(e^{2}, \Delta e^{2}\right)$-FIS outperform the systems with one-input $\left(e^{2}\right)$-FIS. It is noticed that the FLC-LMS with a

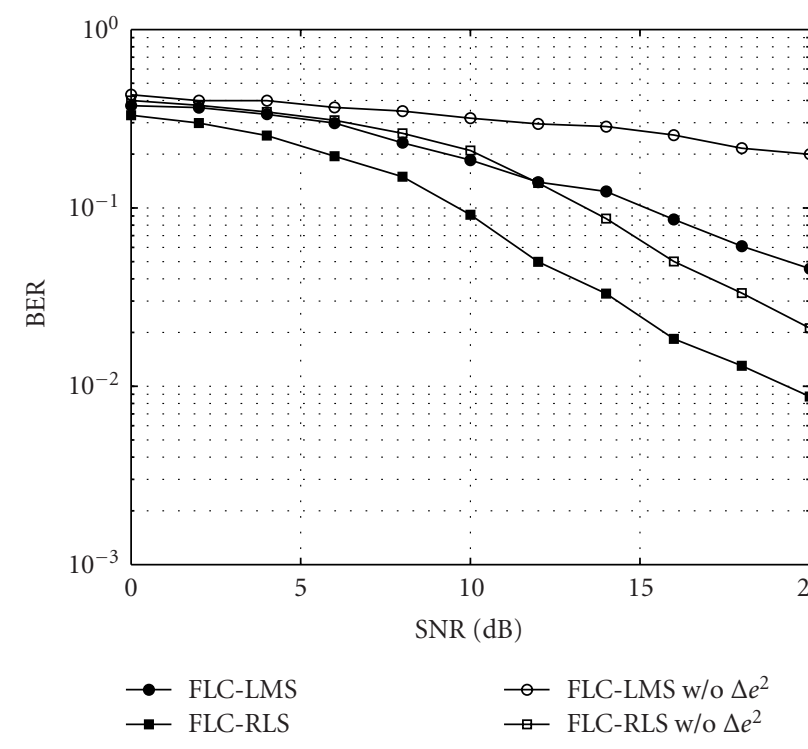

(a)

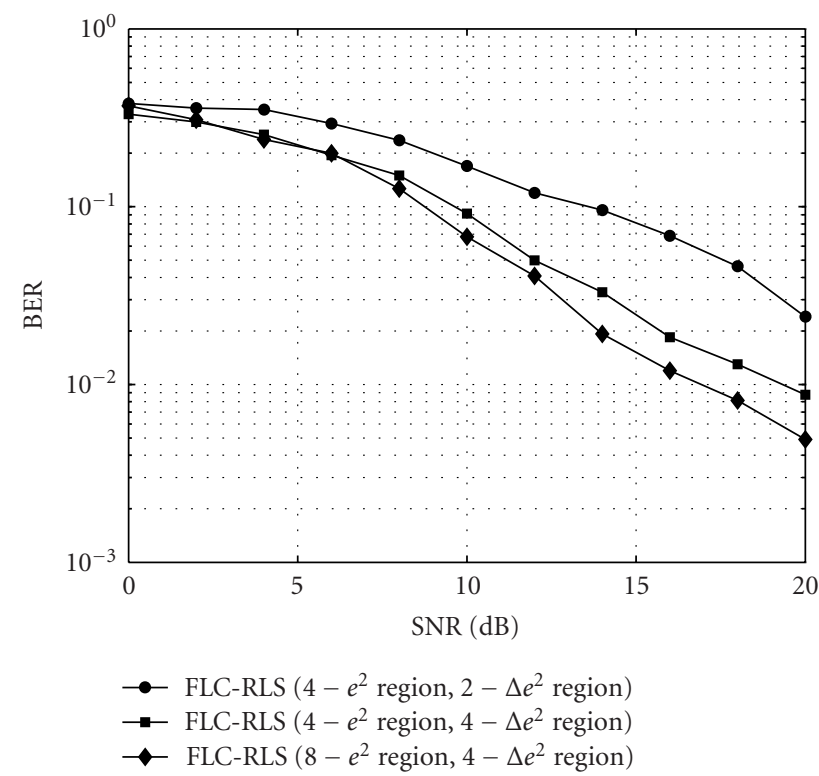

(b)

FIGURE 10: (a) BER performance of the proposed FLC-LMS and FLC-RLS algorithms versus SNR parameterized by the size of the FISs and (b) BER performance of the proposed FLC-RLS algorithm versus SNR parameterized by the size of the $P$-partitioned MBFs, for $K=17\left(K_{\text {intra }}=15, K_{\text {inter }}=2\right), K_{l}=3, M=2$, and a multipath fading rate $=1 / 500$ fade cycle/symbol.

$\left(e^{2}, \Delta e^{2}\right)$-FIS demonstrates a comparable performance in demodulation to the FLC-RLS with a $\left(e^{2}\right)$-FIS. In Figure 10(b), results show that the more regions of the MBFs are partitioned, the better BER performance is achieved. In addition, the improvement in BER performance is enhanced substantially when the larger $P$-partitioned regions to the universe of $\Delta e^{2}$ are employed. These facts imply that the 


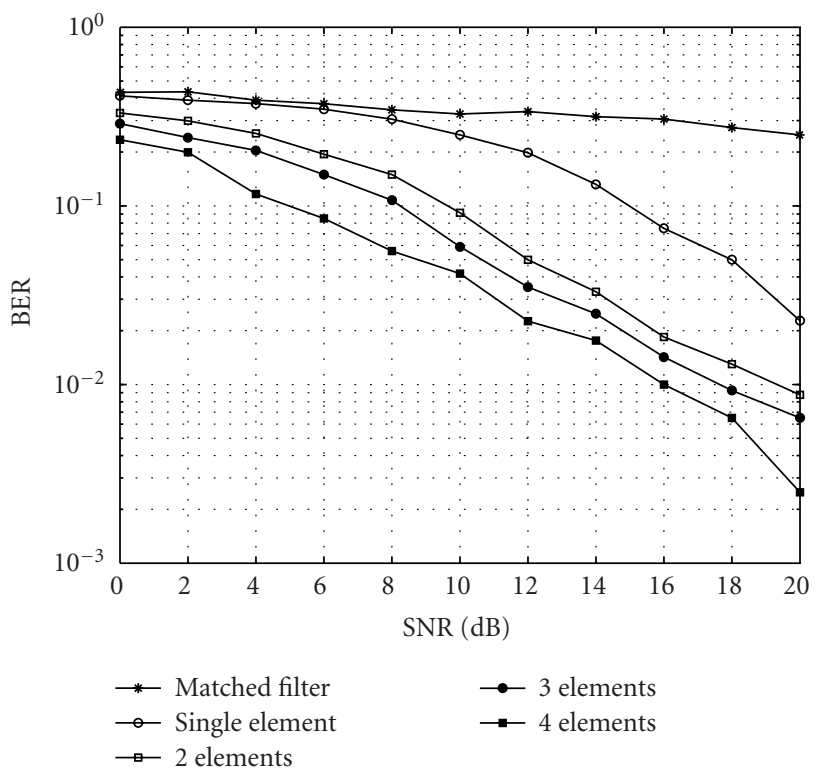

FIGURE 11: BER performance of the proposed FLC-RLS algorithm versus SNR parameterized by the array of the $M$-element receiving antenna for $K=17\left(K_{\text {intra }}=15, K_{\text {inter }}=2\right), K_{l}=3$, and a multipath fading rate $=1 / 500$ fade cycle/symbol.

parameter $\Delta e^{2}$ provides the proposed MMSE receiver with the precise knowledge of the fast-fading channels and enables rapid adaptive convergence.

In Figure 11, the BER performance of the FLC-RLS algorithm is presented in terms of SNR parameterized by the size of the $M$-element receiving antenna array for $K=17$ $\left(K_{\text {intra }}=15, K_{\text {inter }}=2\right), K_{l}=3$, and a multipath fading rate $=1 / 500$ fade cycle/symbol. The proposed FLC-RLS provides superior performance as an increasing function of the size of the $M$-element antenna array. This is made possible because the MAI and ISI between users are suppressed successfully by the proposed algorithm in that they adaptively place nulls in the directions of the stronger interference. An $M$-element beamforming array antenna is known to be able to perform beamforming with $M-1$ degrees of freedom to control the directions of $M-1$ nulls of the antenna. Furthermore, the FLC-RLS array receiver yields a superior performance in demodulation over the conventional receiver that uses a standard matched filter.

Simulation results in Figures 12 and 13(a) demonstrate the BER performance of the FLC-RLS algorithm as a function of SNR parameterized by a variety of FISs for $K=17$ $\left(K_{\text {intra }}=15, K_{\text {inter }}=2\right), K_{l}=3, M=2$, and $N_{T}=2000$ in the presence of the stationary and nonstationary environments, respectively. In a stationary environment, the FLC-RLS systems with two-input FIS (i.e., $\left(e^{2}, N_{T}\right)$ or $\left.\left(e^{2}, \Delta e^{2}\right)\right)$ provide a better BER performance than the FLC-RLS systems with a single input $\left(e^{2}\right)$-FIS, as shown in Figure 12. Notably, it is difficult to evaluate the difference in demodulation achieved by parameters $N_{T}$ and $\Delta e^{2}$. However, the improvement in demodulation is substantial when the proposed FLC-RLS algorithm operates in a nonstationary (i.e., a multipath fading

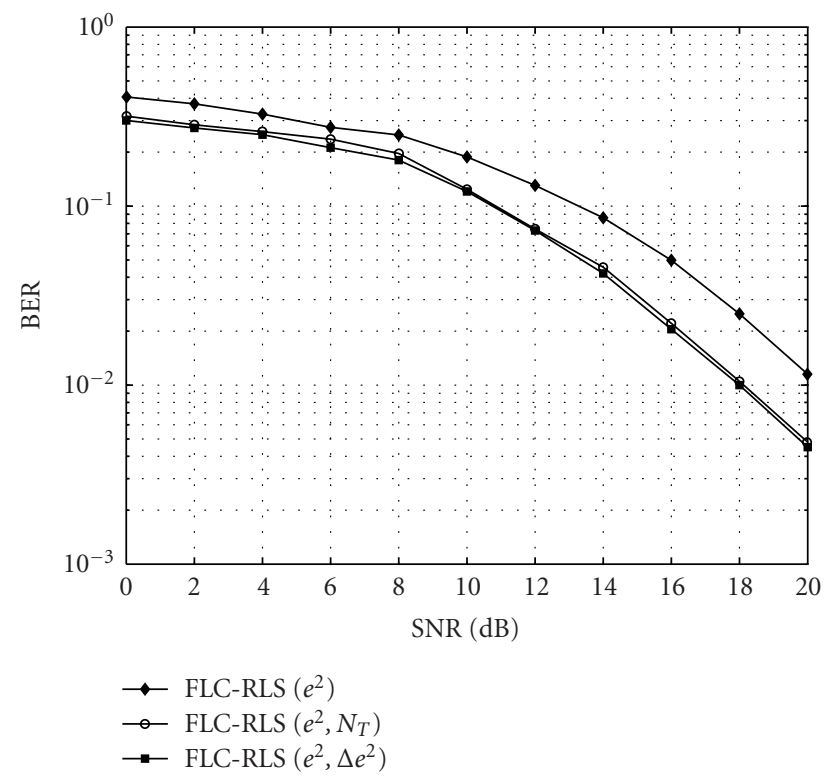

FIGURE 12: BER performance of the proposed FLC-RLS algorithm versus SNR parameterized by a variety of FIS for $K=17$ ( $K_{\text {intra }}=$ $\left.15, K_{\text {inter }}=2\right), K_{l}=3, M=2$, and $N_{T}=2000$ in the presence of the stationary environment.

rate $=1 / 500$ fade cycle/symbol) environment, as illustrated in Figure 13(a). This is because the FLC-RLS algorithm with the $\left(e^{2}, N_{T}\right)$-FIS fails to track effectively the statistical variations of the dynamic fading channels, due to the use of the fixed-length duration of training. Similar results are shown in Figure 13(b) for the convergence/tracking performance when the proposed FLC-RLS algorithm operates in a nonstationary environment with a multipath fading rate $=1 / 200$ fade cycle/symbol. Again, the use of a $\left(e^{2}, \Delta e^{2}\right)$-FIS demonstrates a much better convergence and steady-state characteristic in multipath dynamic fading channels.

\section{CONCLUSIONS}

An adaptive linear MMSE receiver is an effective mean of interference suppression in DS-CDMA systems, but is inapplicable due to the excessively computational complexity. The computation load required to realize the MMSE receiver is formidable when the number of the filter tap-weights for DSCDMA systems is large. Moreover, the slow convergence of an adaptive MMSE receiver is undesirable for the dynamic multipath fading channels. In this paper, a robust adaptive MMSE array receiver based on the fuzzy-inference-based RLS algorithm is developed for space-time joint DS-CDMA interference mitigation in the presence of frequency-selective multipath fading. An alternative lower complexity version of the proposed MMSE linear receiver is developed based on the LMS algorithm with a fuzzy-logic controlled adaptation step size. The incorporation of an FIS into the LMS and RLS approaches produces convergence and steady-state benefits, at the expense of a very slight increase in computational or receiver hardware complexity. Simulations show 


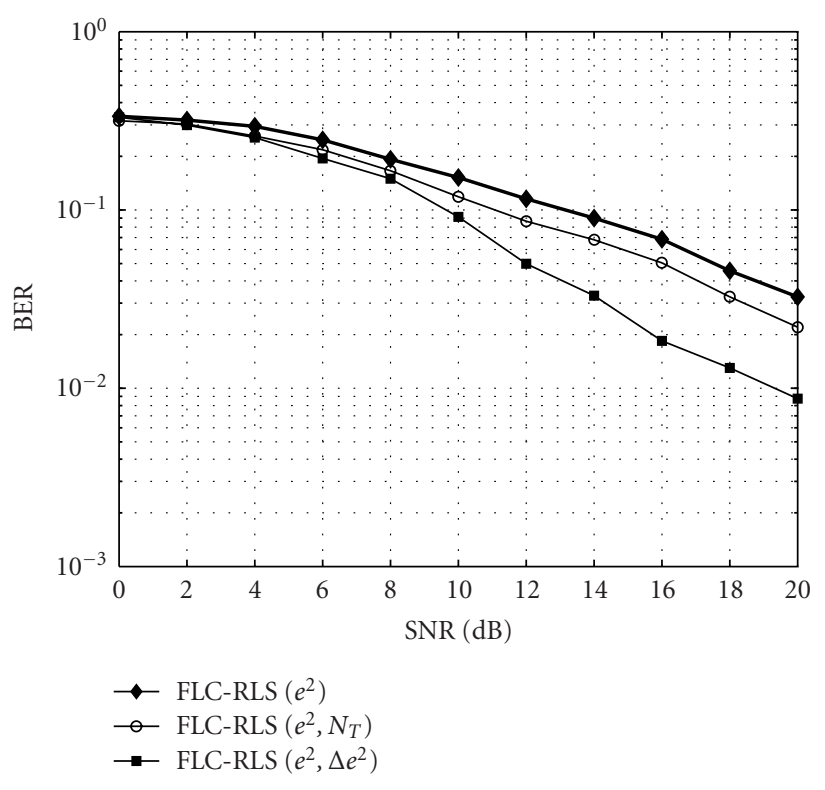

(a)

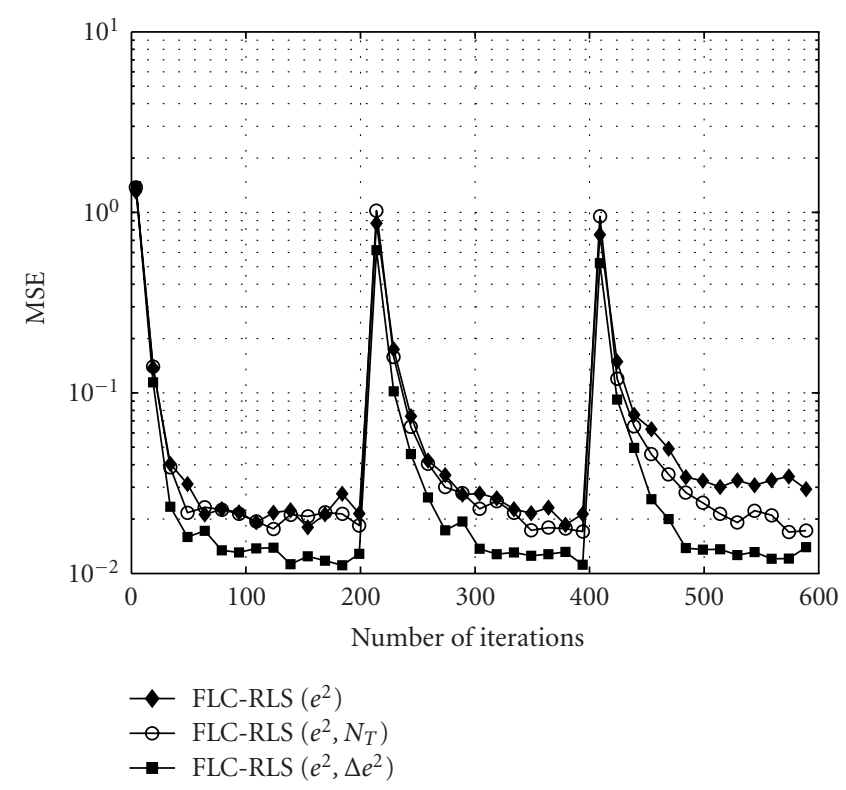

(b)

FIGURE 13: (a) BER performance of the FLC-RLS algorithm versus SNR parameterized by a variety of FISs for a multipath fading rate $=1 / 500$ fade cycle/symbol. (b) Mean square error of the FLC-RLS algorithm versus the number of iterations $L$ parameterized by a variety of FISs for a multipath fading rate $=1 / 200$ fade cycle/symbol and SNR $=20 \mathrm{~dB}$, both cases with the parameters $K=17\left(K_{\text {intra }}=15, K_{\text {inter }}=2\right), K_{l}=3, M=2$, and $N_{T}=2000$.

that the proposed FLC-RLS adaptive receiver for interference suppression provides an extremely fast convergence rate, superior dynamic-tracking capability, and excellent steadystate performance in time-varying channel conditions. Also, results illustrate that an improvement in BER performance is achieved by the fuzzy-inference-based algorithms. Remarkably, the FLC-RLS approach accomplishes $8 \mathrm{~dB}, 4 \mathrm{~dB}$, $2.1 \mathrm{~dB}$, and $1.5 \mathrm{~dB}$ over the C-RLS, OFFF-RLS, GVFF-RLS, and VFF-RLS, respectively, at a fixed BER of $4 \times 10^{-2}$ for multipath fading channels. The FLC-LMS approach outperforms Kwong's and Aboulnasr's VSS-LMS algorithms by about $3 \mathrm{~dB}$ and $6 \mathrm{~dB}$, respectively, at BER $=0.25$. The BER improvement is enhanced substantially when the comparison is performed with the C-LMS and Harris's VSS-LMS algorithms. In addition, the proposed DS-CDMA systems with the use of a $\left(e^{2}\right.$, $\Delta e^{2}$ )-FIS demonstrate a much better performance for data demodulation over DS-CDMA systems with either $\left(e^{2}\right)$-FIS or $\left(e^{2}, N_{T}\right)$-FIS. It has to be stressed that the improvement in demodulation is significantly enhanced when the proposed $\left(e^{2}, \Delta e^{2}\right)$-FIS system is employed instead of the $\left(e^{2}\right)$-FIS or $\left(e^{2}, N_{T}\right)$-FIS system in a time-varying multipath fading environment. Furthermore, the fuzzy-based LMS and RLS receivers obtain a better performance than the C-LMS and CRLS receivers and achieve a substantial improvement in performance as an increasing function of the size of an antenna array or a fuzzy set. As a consequence, a combination of the $\left(e^{2}, \Delta e^{2}\right)$-FIS and LMS/RLS adaptive filtering techniques provides an effective method for applications in real wireless communication systems.

\section{REFERENCES}

[1] Z. Xie, R. T. Short, and C. K. Rushforth, "A family of suboptimum detectors for coherent multiuser communications," IEEE Journal on Selected Areas in Communications, vol. 8, no. 4, pp. 683-690, 1990.

[2] B. Widrow, P. E. Mantey, L. J. Griffiths, and B. B. Goode, "Adaptive antenna systems," Proceedings of the IEEE, vol. 55, no. 12, pp. 2143-2159, 1967.

[3] C. W. Jim, "A comparison of two LMS constrained optimal array structures," Proceedings of the IEEE, vol. 65, no. 12, pp. 1730-1731, 1977.

[4] M. Honig, U. Madhow, and S. Verdú, "Blind adaptive multiuser detection," IEEE Transactions on Information Theory, vol. 41, no. 4, pp. 944-960, 1995.

[5] H. V. Poor and X. Wang, "Code-aided interference suppression for DS/CDMA communications. II. Parallel blind adaptive implementations," IEEE Transactions on Communications, vol. 45, no. 9, pp. 1112-1122, 1997.

[6] V. Kecman, Learning and Soft Computing: Support Vector Machines, Neural Networks, and Fuzzy Logic Models, MIT Press, Cambridge, Mass, USA, 2001.

[7] X. Wu, L. Ge, and G. Liang, "Adaptive power control on the reverse link for CDMA cellular system," in Proceedings of 5th IEEE Asia-Pacific Conference on Communications and 4th Optoelectronics and Communications Conference (APCC/OECC '99), vol. 1, pp. 608-611, Beijing, China, October 1999.

[8] P.-R. Chang and B.-C. Wang, "Adaptive fuzzy power control for CDMA mobile radio systems," IEEE Transactions on Vehicular Technology, vol. 45, no. 2, pp. 225-236, 1996.

[9] P.-R. Chang and B.-C. Wang, "Adaptive fuzzy proportional integral power control for a cellular CDMA system with time delay," IEEE Journal on Selected Areas in Communications, vol. 14, no. 9, pp. 1818-1829, 1996. 
[10] M. Jamshidi, "Fuzzy logic software and hardware," in Fuzzy Logic and Control: Software and Hardware Applications, Prentice-Hall, Englewood cliffs, NJ, USA, 1993.

[11] W.-S. Gan, "Designing a fuzzy step size LMS algorithm," IEE Proceedings - Vision, Image and Signal Processing, vol. 144, no. 5, pp. 261-266, 1997.

[12] R. W. Harris, D. M. Chabries, and F. A. Bishop, "A variable step (VS) adaptive filter algorithm," IEEE Transactions Acoustics, Speech, Signal Processing, vol. 34, no. 2, pp. 309-316, 1986.

[13] R. H. Kwong and E. W. Johnston, "A variable step size LMS algorithm," IEEE Transactions on Signal Processing, vol. 40, no. 7, pp. 1633-1642, 1992.

[14] S. Song, J.-S. Lim, S. J. Back, and K.-M. Sung, "Variable forgetting factor linear least squares algorithm for frequency selective fading channel estimation," IEEE Transactions on Vehicular Technology, vol. 51, no. 3, pp. 613-616, 2002.

[15] J. G. Proakis, Digital Communications, McGraw-Hill, New York, NY, USA, 4th edition, 2000.

[16] M. L. Honig, S. L. Miller, M. J. Shensa, and L. B. Milstein, "Performance of adaptive linear interference suppression in the presence of dynamic fading," IEEE Transactions on Communications, vol. 49, no. 4, pp. 635-645, 2001.

[17] M. L. Honig and J. S. Goldstein, "Adaptive reduced-rank interference suppression based on the multistage Wiener filter," IEEE Transactions on Communications, vol. 50, no. 6, pp. 986994, 2002.

[18] P. B. Rapajic and B. S. Vucetic, "Adaptive receiver structures for asynchronous CDMA systems," IEEE Journal on Selected Areas in Communications, vol. 12, no. 4, pp. 685-697, 1994.

[19] M. Abdulrahman, A. U. H. Sheikh, and D. D. Falconer, "Decision feedback equalization for CDMA in indoor wireless communications," IEEE Journal on Selected Areas in Communications, vol. 12, no. 4, pp. 698-706, 1994.

[20] U. Madhow and M. L. Honig, "MMSE interference suppression for direct-sequence spread-spectrum CDMA," IEEE Transactions on Communications, vol. 42, no. 12, pp. 31783188, 1994.

[21] S. Haykin, Adaptive Filter Theory, Prentice-Hall, Englewood Cliffs, NJ, USA, 3rd edition, 1996.

[22] L. A. Zadeh, "Fuzzy sets," Information and Control, vol. 8, no. 3, pp. 338-353, 1965.

[23] Y.-L. Chen, Y.-S. Lin, J.-H. Wen, W.-M. Chang, and J. Liao, "Combined fuzzy-based rate and selective power control in multimedia CDMA cellular systems," in Proceedings of 58th IEEE Vehicular Technology Conference (VTC '03), vol. 4, pp. 2506-2510, Orlando, Fla, USA, October 2003.

[24] M. J. Patyra, J. L. Grantner, and K. Koster, "Digital fuzzy logic controller: design and implementation," IEEE Transactions on Fuzzy Systems, vol. 4, no. 4, pp. 439-459, 1996.

[25] A. Costa, A. De Gloria, P. Faraboschi, A. Pagni, and G. Rizzotto, "Hardware solutions for fuzzy control," Proceedings of IEEE, vol. 83, no. 3, pp. 422-434, 1995.

[26] T. Aboulnasr and K. Mayyas, "A robust variable step-size LMStype algorithm: analysis and simulations," IEEE Transactions on Signal Processing, vol. 45, no. 3, pp. 631-639, 1997.

[27] J. Lin, J. G. Proakis, F. Ling, and H. Lev-Ari, "Optimal tracking of time-varying channels: a frequency domain approach for known and new algorithms," IEEE Journal on Selected Areas in Communications, vol. 13, no. 1, pp. 141-154, 1995.

[28] W. C. Jakes, Microwave Mobile Communications, John Wiley \& Sons, New York, NY, USA, 1974.
[29] C. F. So, S. C. Ng, and S. H. Leung, "Gradient based variable forgetting factor RLS algorithm," Signal Processing, vol. 83, no. 6, pp. 1163-1175, 2003.

[30] D. K. Borah and B. T. Hart, "Frequency-selective fading channel estimation with a polynomial time-varying channel model," IEEE Transactions on Communications, vol. 47, no. 6 , pp. 862-873, 1999.

Chia-Chang $\mathrm{Hu}$ received the B.S. and M.S. degrees from the National Cheng Kung University, Tainan, Taiwan, in 1990 and 1992, respectively, and the Ph.D. degree from the University of Southern California, Los Angeles, Calif, in 2002, all in electrical engineering. Since February 2003, he has been with the Department of Electrical Engineering, the National Chung Cheng University, Chia-Yi, Taiwan, where he is an Assistant

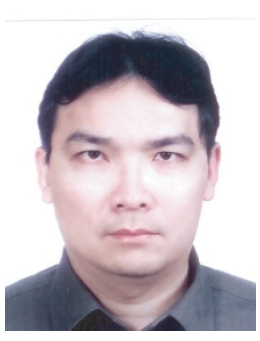
Professor. His current research interests are in the areas of communication theory and advanced signal processing for communications, with a special emphasis on statistical signal and array processing, wireless multiuser communications, synchronization, blind channel equalization, wideband CDMA, and ultra-wideband technology.

Hsuan-Yu Lin received the B.S. and M.S. degrees in electrical engineering from the National Chung Cheng University (CCU), Chia-Yi, Taiwan, in 1996 and 1998, respectively. He is currently pursuing the Ph.D. degree at CCU, where he works on adaptive signal processing and its applications in mobile communications. In addition, he is a leading engineer at TaiwanMobile, where he works in the area of $3 \mathrm{G}$ system design and

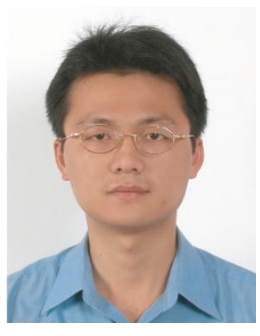
performance optimization. His research interests include adaptive signal processing, radio resource management, and performance evaluation of cellular systems.

Yu-Fan Chen was born in Taiwan, the Republic of China, on 18 August 1981. He received the M.S. degree in communication engineering from the National Chung Cheng University (CCU), Chia-Yi, Taiwan, in 2005. He is currently pursuing the Ph.D. degree at the Department of Communication Engineering, the National Chiao Tung University (NCTU), Hsinchu, Taiwan. His research interest is in the area of advanced signal processing for wireless communications.

Jyh-Horng Wen received the Ph.D. degree in electrical engineering from the National Taiwan University, Taipei, in 1990. From 1983 to 1991, he was a Research Assistant with the Institute of Nuclear Energy Research, Taoyun, Taiwan. Since February 1991, he has been with the Institute of Electrical Engineering, National Chung Cheng University, Chia-Yi, Taiwan, first as an Associate Professor and, since 2000, as a Profes$(2=1$ sor. He was also the Managing Director of the Center for Telecommunication Research, National Chung Cheng University, from 
August 2000 to July 2004. Currently, he is also the Dean of General Affairs, National Chi Nan University. He is an Associate Editor of the Journal of the Chinese Grey System Association. His current research interests include computer communication networks, cellular mobile communications, personal communications, spreadspectrum techniques, wireless broadband systems, and gray theory. He is a Member of the IEEE Communication Society, the IEEE Vehicular Technology Society, the International Association of Science and Technology for Development, the Chinese Grey System Association, and the Chinese Institute of Electrical Engineering. 